\title{
OPTIMUM DESIGN OF FLEXTENSIONAL PIEZOELECTRIC ACTUATORS INTO TWO SPATIAL DIMENSIONS*
}

\author{
R. AMIGO $^{\dagger}$, S. M. GIUSTI ${ }^{\ddagger}$, A. A. NOVOTNY ${ }^{\S}$, E. C. N. SILVA ${ }^{\dagger}$, AND J. SOKOŁOWSKI
}

\begin{abstract}
Piezoelectric actuators are in common use for control of distributed parameter systems. We consider the topology optimization of a multiphysic model in piezoelectricity into two spatial dimensions. The topological derivative of a tracking-type shape functional is derived in its closed form for the purpose of shape optimization of piezoelectric actuators. The optimum design procedure is applied to a micromechanism which transforms the electrical energy supplemented via its piezoceramic part into the elastic energy of an actuator. The domain decomposition technique and the Steklov-Poincaré pseudodifferential boundary operator are employed in the asymptotic analysis of the shape functional defined on a part of the boundary of the elastic body under consideration. The new method of sensitivity analysis is general and can be used for shape-topological optimization in a broad class of multiphysics models. Our numerical results confirm the efficiency of the proposed approach to optimum design in multiphysics.
\end{abstract}

Key words. asymptotic analysis, Steklov-Poincaré operator, Dirichlet-to-Neumann map, domain decomposition, topological derivative, piezoelectric actuator, topology design

AMS subject classifications. 35J30, 49Q10, 49Q12, 74K20, 74P15

DOI. $10.1137 / 151004860$

1. Introduction. In this paper we are interested in the optimal design of piezoelectric actuators, which consist of multiflexible structures actuated by piezoceramic devices that generate an output displacement in a specified direction on the boundary of the actuated part $[6,30]$. The multiflexible structure transforms the piezoceramic output displacement by amplifying and changing its direction. This kind of mechanism can be manufactured at a very small scale. Therefore, the spectrum of applications of such microtools has become broader in recent years, including microsurgery, nanotechnology processing, and cell manipulation, among others. Yet, the development of microtools requires the design of actuated multiflexible structures which are able to produce complex movements originating from simple expansion/contraction of the piezoceramic actuator. The performance of microtools can be strongly enhanced by optimizing the actuated multiflexible structures with respect to their shape and their topology $[7,8,9]$. The shape sensitivity analysis of such coupled models has been fully developed in [20] and [19] for quasi-electrostatic layered piezoelectric devices and for nonstationary elastic, piezoelectric, and acoustic coupled systems, respectively. For

${ }^{*}$ Received by the editors January 21, 2015; accepted for publication (in revised form) December 28, 2015; published electronically March 24, 2016. This research was partly supported by the CNPq (Brazilian Research Council), CAPES (Brazilian Higher Education Staff Training Agency), and FAPERJ (Research Foundation of the State of Rio de Janeiro), and by ANR-12-BS0-0007 Optiform. http://www.siam.org/journals/sicon/54-2/100486.html

${ }^{\dagger}$ Department of Mechatronics and Mechanical Systems Engineering, University of São Paulo, 2231 - 05508-030, São Paulo, Brazil (ricardo.amigo@usp.br, ecnsilva@usp.br). The work of the fourth author was supported in part by the FAPESP (Research Foundation of the State of São Paulo), and by the CNPq under grant 304121/2013-4.

‡Universidad Tecnológica Nacional (UTN/FRC) - CONICET, Maestro M. López esq. Cruz Roja Argentina, X5016ZAA - Córdoba, Argentina (sgiusti@civil.frc.utn.edu.ar).

${ }_{\S}$ Corresponding author. Laboratório Nacional de Computação Científica LNCC/MCT, 25651-075 Petrópolis - RJ, Brazil (novotny@lncc.br).

I Université de Lorraine, CNRS, INRIA, Institute Élie Cartan Nancy, UMR7502, BP 239-54506 Vandoeuvre Lès Nancy Cedex, France, and Systems Research Institute of the Polish Academy of Sciences, 01-447 Warsaw, Poland (jan.sokolowski@univ-lorraine.fr). 
the mathematical theory concerning coupled PDEs systems, the reader may refer to, e.g., $[18,21,22]$.

A general approach to dealing with shape and topology optimization design is based on the topological derivative. In fact, this relatively new concept represents the first term of the asymptotic expansion of a given shape functional with respect to the small parameter which measures the size of singular domain perturbations, such as holes, inclusions, source-terms, and cracks. The topological asymptotic analysis was introduced in the fundamental paper [31] and has been successfully applied in the treatment of problems such as topology optimization [5], inverse analysis [16], image processing [15], multiscale constitutive modeling [4], fracture mechanics sensitivity analysis [13], and damage evolution modeling [1]. For an account of new developments in this branch of shape optimization, we refer the reader to [27].

In particular, here the topological derivative is applied in the context of topology optimization of piezoelectric actuated multiflexible structures into two spatial dimensions. The basic idea consists of maximizing the performance of the microtool by introducing small inclusions into the multiflexible elastic part. Since this problem is modeled by a coupled electromechanical system, the domain decomposition technique combined with the Steklov-Poincaré pseudodifferential boundary operator is used to derive the first-order term of the asymptotic expansion of the shape functional with respect to the small parameter measuring the size of the inclusions. Thus, a new method of topological sensitivity analysis is proposed for the coupled models. In our framework the topological derivatives for the tracking-type functionals are obtained in their closed forms, which can be used, e.g., as a steepest descent direction in the design of microtools using the topology optimization method. However, in shape optimization with PDE constraints there are three main issues to be solved:

- existence of optimal shapes,

- necessary optimality conditions,

- numerical methods.

The existence of optimal shapes can be assured by regularity conditions imposed on the boundaries of admissible domains. Without such restrictive constraints on admissible shapes, the existence issue cannot be solved in general. The special structure of the shape optimization problem can be used for the direct proof of the existence, e.g., by the application of Mosco convergence to the elliptic problems. Unfortunately, even in such a situation, the results obtained are not in general constructive.

Necessary optimality conditions are obtained by shape sensitivity analysis. Recently, asymptotic analysis has been employed in the context of singular boundary perturbations in order to obtain the topological derivatives of shape functionals for elliptic boundary value problems. These kinds of results are also obtained for our problem. The knowledge of the shape gradients and the topological derivatives for a specific shape functional is required in order to formulate the necessary optimality conditions as well as to devise numerical methods of shape optimization. There is also the specific structure of shape derivatives obtained for shape differentiable functionals in the form of distributions supported on the moving boundaries [34]. The structure should be taken into account when using the discretization of a continuous gradient combined with the standard methods of nonlinear optimization like gradient or Newton methods in the numerical context.

In order to fix these ideas, let us consider a geometrical domain $\Omega$ and its singularly perturbed counterpart $\Omega_{\varepsilon}=\Omega \backslash \overline{B_{\varepsilon}}$ obtained by the nucleation of a small hole $B_{\varepsilon}=$ $\{|x-\widehat{x}|<\varepsilon\}$ with center at an arbitrary point $\widehat{x}$ of $\Omega$. There are two different 
expansions of

$$
\varepsilon \mapsto j(\varepsilon):=J\left(\Omega_{\varepsilon}\right)
$$

The first one is obtained by the classical shape sensitivity analysis for $\varepsilon>0$, namely

$$
j(\varepsilon+\delta)=j(\varepsilon)+\delta j^{\prime}(\varepsilon)+o(\varepsilon ; \delta) .
$$

The second expansion is obtained by asymptotic analysis in singularly perturbed domains for $\varepsilon=0^{+}$, that is,

$$
j(\varepsilon)=j(0)+f(\varepsilon) \mathcal{T}(\widehat{x})+o(f(\varepsilon)) .
$$

The topological derivative $\widehat{x} \mapsto \mathcal{T}(\widehat{x})$ can be used at the preliminary step of an optimization procedure to detect the location and the number of small holes inserted into $\Omega$ in order to improve the value of the shape functional. Therefore, a robust formula for the topological derivative is required for the precise performance of this step of the procedure. This goal is achieved in this paper for the piezo model under consideration, using the appropriate adjoint state equation.

It turns out that the nature of the Taylor expansion of the shape functional $\varepsilon \mapsto J\left(\Omega_{\varepsilon}\right)$ with respect to $\varepsilon \in\left[0, \varepsilon_{0}\right), \varepsilon_{0}>0$, evaluated in the singularly perturbed domain $\Omega_{\varepsilon}$, depends on the boundary conditions of the state equation prescribed on the boundaries $\partial B_{\varepsilon}=\{|x-\widehat{x}|=\varepsilon\}$. The direct derivation of the one-term asymptotic expansion for (1.1) at $\varepsilon=0^{+}$leads to the self-adjoint extensions of elliptic operators [26]. The appropriate adjoint state combined with the polarization tensors is introduced in order to obtain the appropriate representation of the topological derivative for numerical methods of shape optimization. The latter step in this procedure is complicated for the coupled models. Therefore, in this paper, a general method is proposed in order to overcome this difficulty, following the original ideas presented in [32]. It consists of decomposing the topologically perturbed geometrical domain into subdomains with different physical properties. The mutual influence of the subdomains is affected by the transmission conditions on the interfaces. In other words, the fields of mechanical and electric natures are coupled by the transmission conditions, as well as by the mathematical models in the interior of each subdomain. This means that in the elastic material of the body the fictitious ring domain $C(R, \varepsilon):=\{\varepsilon<|x-\widehat{x}|<R\}$ is introduced for the purpose of the asymptotic analysis with respect to $\varepsilon \rightarrow 0$, and the result of the analysis is expressed on the boundary of the ball $B_{R}=\{|x-\widehat{x}|<R\}$. From the asymptotic expansions of elastic energy in the interior of $B_{R}$ or $C(R, \varepsilon)$ the expansion of the Dirichlet-to-Neumann map associated with the ball or with the ring is obtained. Once we have the asymptotic expansion in hand, the Dirichlet-to-Neumann operator in $B_{R}$ is employed as the Steklov-Poincaré boundary pseudodifferential operator in the truncated domain $\Omega_{R}:=\Omega \backslash \overline{B_{R}}$. In this way, the influence of singularities associated with the limit passage $\varepsilon \rightarrow 0$ is modeled in the truncated domain via nonlocal boundary conditions, and the subdomain $B_{R}$ is eliminated from the analysis. Otherwise, the asymptotic analysis of the coupled model in the singularly perturbed geometrical domain should be performed, which would not be the best idea because of the complexity of such an approach. Finally, the domain decomposition method combined with the asymptotic expansions in the ring associated with the small parameter $\varepsilon \rightarrow 0$ allows us to find the topological derivative of the shape functional defined in the truncated domain $\Omega \backslash \overline{B_{R}}$ for all $R>\varepsilon \rightarrow 0$. This approach simplifies the topological asymptotic analysis of the shape functional under consideration. See a sketch in Figure 1. 


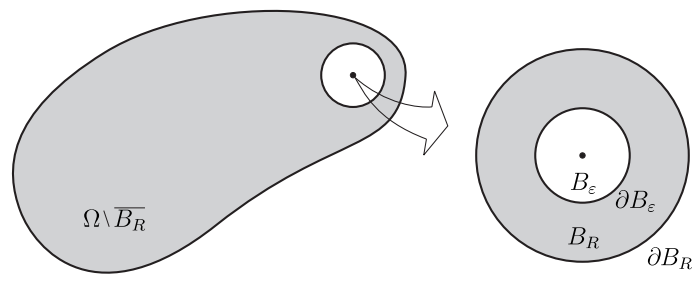

FIG. 1. A truncated domain.

Remark 1.1. There is a double notation for the same boundary pseudodifferential operator on $\partial B_{R}$ depending on whether $\partial B_{R}$ is considered as the exterior boundary of the fictitious subdomain $B_{R}$ or the interior boundary of truncated domain $\Omega_{R}$. More precisely, the nonlocal boundary operator stands for the Steklov-Poincaré operator when acting on the interior boundary $\partial B_{R}$ of the truncated domain $\Omega_{R}$. Thus, the elasticity boundary value problem in $B_{R}$ is called the interior problem of fictitious domain decomposition, and the coupled model in $\Omega_{R}$ is called the exterior problem of fictitious domain decomposition in the notation employed in the paper. The boundary $\partial B_{R}$ is used as an interface of the fictitious domain decomposition introduced exclusively for the purpose of asymptotic analysis in the singularly perturbed domain.

The paper is organized as follows. In section 2 the Steklov-Poincaré pseudodifferential boundary operator is introduced. The electromechanical coupled system modeling the piezoelectric actuators, as well as the adopted shape functional, are presented in section 3. The associated topological asymptotic expansion is rigorously derived in section 4. In particular, the topological derivatives in their closed forms associated with inclusions and holes are obtained for two and three spatial dimensions [27]. In section 6 some numerical experiments of topology optimization of piezoelectric actuators are presented. Finally, some concluding remarks and perspectives are given in section 7 .

2. Steklov-Poincaré and Dirichlet-to-Neumann boundary operators. In order to perform the shape-topological sensitivity analysis of a coupled model defined in $\Omega$, in the presence of singular domain perturbations resulting from the insertion of cavities, holes, or inclusions into the elastic subdomain, the fictitious domain decomposition $\Omega:=\Omega_{R} \cup \overline{B_{R}}$ is introduced into the model. The small region $B_{R}$, which includes the singular domain perturbation $B_{\varepsilon}$, is selected for the asymptotic analysis performed, e.g., by the method of compound asymptotic expansions for the singularity depending on the small parameter $\varepsilon \rightarrow 0$. In this way the asymptotic analysis is performed in a simple geometry with radial symmetry of the ball $B_{R}$, and it is separated from the shape-topological sensitivity analysis of the functional which is performed in $\Omega_{R}$ with the nonlocal boundary conditions defined by the SteklovPoincare operator on $\partial B_{R}$, and in the absence of the singularity $B_{\varepsilon}$ inside of the domain.

The asymptotic expansion of Dirichlet-to-Neumann boundary operators with respect to $\varepsilon \rightarrow 0$ is performed for the boundary value problem of linear elasticity defined in the ring $C(R, \varepsilon)$. The case of an inclusion $B_{\varepsilon}$ in $B_{R}$ can be considered as a regular perturbation of the bilinear form associated with the boundary value problem in $\Omega_{R}$, depending on the contrast parameter $0<\gamma<\infty$. The limit case obtained for $\gamma \rightarrow 0$ with the inclusion which is replaced by a hole or a cavity of the radius $\varepsilon \rightarrow 0$ is considered in $C(R, \varepsilon)$ as the singular perturbation of $B_{R}$. 
Thus, following [33], we present the detailed asymptotic analysis of the representative singular domain perturbation using the insertion of a hole in two spatial dimensions. The case of an inclusion can be analyzed in the same manner by using the Kolosov complex potentials in two spatial dimensions. The asymptotic analysis in three spatial dimensions can be performed by an application of the method of compound asymptotic expansions. In particular, we introduce the Steklov-Poincaré pseudodifferential boundary operator obtained explicitly from the closed solutions of the linear elasticity boundary value problems.

Remark 2.1. The asymptotic analysis of linear elasticity systems in a truncated domain is performed in [12] using an application of the Green's function. In particular, the statement on a spherical hole can be found in section 3.3, page 1766. In contrast to [12], the method developed in [32] has been designed for the purpose of asymptotic analysis in singularly perturbed domains for a class of nonlinear elasticity systems. It relies on the knowledge of the explicit solution of the elasticity problem in a subdomain of simple geometry. The explicit solution in the ring in two spatial dimensions is obtained by the complex Kolosov potentials [23]. By a result from the functional analysis on positive, self-adjoint operators, the expansion of the elastic energy in the ring gives rise to the expansion of the Steklov-Poincaré operator on the boundary of the topologically perturbed truncated domain, with the remainder uniformly bounded in the operator norm. In addition, the explicit solution allows us to replace the expression of the topological derivative unbounded in the energy norm by its equivalent bounded in the energy norm. In this way the truncated domain technique proposed in [12] was extended to the nonlinear contact problems in elasticity fully developed in [32]. See also [26] for the general case of elliptic systems and for the self-adjoint extensions of elliptic operators in nontruncated domains. In this paper the method derived in [32] is now applied in the context of a coupled electromechanical system. We note, however, that the general framework for the topological asymptotic analysis developed in [12] can be applied to coupled systems as well.

2.1. Unperturbed domain. Let us introduce the notation $\Gamma_{R}:=\partial B_{R}$. We use the standard notation for the Sobolev spaces and the linear elasticity boundary value problems $[11,35]$. Let us consider an open bounded domain $\Omega \subset \mathbb{R}^{d}, d=2,3$, and let $\Omega_{R}=\Omega \backslash \overline{B_{R}}$ represent an elastic body, where $B_{R}=\{|x-\widehat{x}|<R\}$ is a ball of radius $R>0$ and centered at the point $\widehat{x} \in \Omega$ such that $B_{R} \subset \Omega$. The Sobolev spaces associated with the weak variational formulation of the linear elasticity boundary value problems are denoted by $H^{1}\left(B_{R}\right):=H^{1}\left(B_{R} ; \mathbb{R}^{d}\right)$ and $H_{0}^{1}\left(B_{R}\right):=H_{0}^{1}\left(B_{R} ; \mathbb{R}^{d}\right)$ for simplicity. The stress tensor is written in the Voigt notation $\sigma(u)=C \nabla^{s} u$, with $C$ the Hooke's tensor of elastic constants, and the bilinear form of linear elasticity is defined on $H_{0}^{1}\left(B_{R}\right)$,

$$
a(u, v)=\int_{B_{R}} \sigma(u) \cdot \nabla^{s} v \quad \forall u, v \in H^{1}\left(B_{R}\right) .
$$

Now we are in position to define the Dirichlet-to-Neumann map in $B_{R}$ given by the solution of an auxiliary, nonhomogeneous Dirichlet boundary value problem in the ball $B_{R}$. Such a map is considered as a pseudodifferential operator on $\Gamma_{R}:=\partial B_{R}$, and it is called the Steklov-Poincaré operator when it is applied to the boundary value problem in the truncated domain for which $\Gamma_{R}$ constitutes a part of the boundary. The truncated domain is used in the framework of the domain decomposition technique for the elasticity problem under consideration. Therefore, we introduce the auxiliary, nonhomogeneous Dirichlet boundary value problem of linear elasticity defined in $B_{R}$, 
namely

$$
u=\varphi \text { on } \Gamma_{R}, \quad u \in H^{1}\left(B_{R}\right): a(u, v)=0 \quad \forall v \in H_{0}^{1}\left(B_{R}\right),
$$

where $\varphi \in H^{1 / 2}\left(\Gamma_{R}\right):=H^{1 / 2}\left(\Gamma_{R} ; \mathbb{R}^{d}\right)$ is given. With the solution $u \in H^{1}\left(B_{R}\right)$ of problem $(2.2)$ is associated its Neumann trace $T(u) \in H^{-1 / 2}\left(\Gamma_{R}\right):=H^{-1 / 2}\left(\Gamma_{R} ; \mathbb{R}^{d}\right)$ such that the Green's formula is valid,

$$
a(u, v)+(T(u), v)_{\Gamma_{R}}=0, \quad u, v \in H^{1}\left(B_{R}\right),
$$

since there is no source in $B_{R}$. By taking into account the nonhomogeneous Dirichlet condition, the Green's formula for the solution of (2.2) becomes

$$
a(u, v)=-(T(\varphi), v)_{\Gamma_{R}} \quad \forall v \in H^{1}\left(B_{R}\right) .
$$

The Dirichlet-to-Neumann map $\mathcal{A}: H^{1 / 2}\left(\Gamma_{R}\right) \mapsto H^{-1 / 2}\left(\Gamma_{R}\right)$ is well defined,

$$
(\mathcal{A}(\varphi), \varphi)_{\Gamma_{R}}:=-(T(\varphi), \varphi)_{\Gamma_{R}} \equiv a(u, u) .
$$

Thus the Steklov-Poincaré operator is defined on $\Gamma_{R}$ by the relation

$$
(\mathcal{A}(\varphi), \varphi)_{\Gamma_{R}}:=a(u, u),
$$

where $\varphi \mapsto(\mathcal{A}(\varphi), \varphi)_{\Gamma_{R}}$ is a symmetric and coercive bilinear form on the space of traces $H^{1 / 2}\left(\Gamma_{R}\right)$.

Now we are going to recall some results of [33] on the asymptotic expansion of the Steklov-Poincaré operator for the singular perturbations of a ring, i.e., in the limit case of $\gamma \rightarrow 0$ and for the interior radius of the ring which tends to zero. This means that in such a case of singular perturbations the inclusion $\omega_{\varepsilon}$ becomes a hole. The case of an inclusion for $0<\gamma<\infty$ is considered as a regular perturbation in coefficients of the elliptic operators, and it can be analyzed from the point of view of asymptotic analysis in a similar way as presented in [27].

Remark 2.2. In the continuous formulation of the shape optimization problem we use the singular perturbations technique and establish the topological derivative of shape functional for nucleation of holes in the elastic subdomain. On the other hand, the numerical algorithm used in the paper is reversible in the sense that we employ very weak material to mimic the holes. We discretize the continuous topological derivatives for the inclusions and have the fixed reference domain during the topology optimization numerical procedure. In this way we avoid the complicated formulae for the topological derivatives of ligaments; see, e.g., [24, 25].

2.2. Singular domain perturbations. The Steklov-Poincaré operator on $\Gamma_{R} \subset$ $\partial \Omega_{R}$ is explicitly constructed by a complex variable method which furnishes the solution of a nonhomogeneous Dirichlet problem in the ring $C(R, \varepsilon)$. For the topological sensitivity analysis we need to establish precise properties of solutions parameterized by small parameter $\varepsilon \rightarrow 0$.

Let $\varepsilon \rightarrow 0$ be small parameter which governs the singular perturbation of the disk $B_{R}$ by insertion of the hole $B_{\varepsilon}$. The resulting domain

$$
\varepsilon \mapsto C(R, \varepsilon):=B_{R} \backslash \overline{B_{\varepsilon}}=\{0 \leq \varepsilon<|x-\widehat{x}|<R\}
$$

is the ring with the fixed boundary $\Gamma_{R}$ and the moving boundary $\varepsilon \mapsto \partial B_{\varepsilon}$. The Dirichlet data $\varphi$ is given on $\Gamma_{R}$, and the homogeneous Neumann boundary condition 
is assumed on $\partial B_{\varepsilon}$ for $\varepsilon>0$. Thus, the variational formulation of the elasticity boundary value problem in the ring

$$
u_{\varepsilon} \in \mathcal{V}+\{\varphi\}: a\left(C(R, \varepsilon) ; u_{\varepsilon}, v\right)=0 \quad \forall v \in \mathcal{V}
$$

admits the unique weak solution, with $\mathcal{V}$ used to denote the space of admissible variations defined in $C(R, \varepsilon)$. Note that here the elliptic regularity applies for $C^{\infty}$ domains $C(R, \varepsilon)$. The Green's formula for a smooth solutions reads

$$
a\left(C(R, \varepsilon) ; u_{\varepsilon}, v\right):=\int_{C(R, \varepsilon)} \sigma\left(u_{\varepsilon}\right) \cdot \nabla^{s} v=\int_{\Gamma_{R}} \sigma\left(u_{\varepsilon}\right) n \cdot v .
$$

If the test function is replaced by the smooth solution, we get the energy equality

$$
a\left(C(R, \varepsilon) ; u_{\varepsilon}, u_{\varepsilon}\right)=\int_{\Gamma_{R}} \sigma\left(u_{\varepsilon}\right) n \cdot \varphi
$$

For weak solutions $u_{\varepsilon} \in H^{1}(C(R, \varepsilon))$ with Dirichlet data $\varphi \in H^{1 / 2}\left(\Gamma_{R}\right)$ the energy equality becomes

$$
a\left(C(R, \varepsilon) ; u_{\varepsilon}, u_{\varepsilon}\right)=\left\langle\sigma\left(u_{\varepsilon}\right) n, \varphi\right\rangle_{\Gamma_{R}}
$$

with the duality pairing $H^{-1 / 2}\left(\Gamma_{R}\right) \times H^{1 / 2}\left(\Gamma_{R}\right)$ on the right-hand side. The associated boundary pseudodifferential operator $\mathcal{A}_{\varepsilon}: \varphi \mapsto \sigma\left(u_{\varepsilon}\right) n$ is symmetric, positive, and self-adjoint, and it is uniquely determined from the energy equality in the ring,

$$
\left\langle\mathcal{A}_{\varepsilon}(\varphi), \varphi\right\rangle_{\Gamma_{R}}:=a\left(C(R, \varepsilon) ; u_{\varepsilon}, u_{\varepsilon}\right) .
$$

In addition, the asymptotic properties of Dirichlet-to-Neumann map

$$
\mathcal{A}_{\varepsilon}: H^{1 / 2}\left(\Gamma_{R}\right) \ni \varphi \mapsto \sigma\left(u_{\varepsilon}\right) n \in H^{-1 / 2}\left(\Gamma_{R}\right)
$$

can be determined by using the complex Kolosov potentials in two dimensional elasticity. The same mapping is used as a nonlocal boundary Steklov-Poincaré operator on $\Gamma_{R} \subset \partial \Omega_{R}$ in the truncated domain $\Omega_{R}$.

Let us observe that, in view of (2.23), the asymptotic expansion of $\varepsilon \mapsto \mathcal{A}_{\varepsilon}$ in the operator norm $\mathcal{L}\left(H^{1 / 2}\left(\Gamma_{R}\right) ; H^{-1 / 2}\left(\Gamma_{R}\right)\right)$ of the symmetric and positive SteklovPoincaré operator $(2.13)$ can be directly obtained from the known asymptotics of the elastic energy in the ring. These asymptotics follow from the explicit solution to the Dirichlet problem in the ring, which can obtained by complex variable technique [14]. This important construction for asymptotic analysis is now presented in detail. Namely, the obtained estimates are now translated into the following theorem concerning the solution of the elasticity system in the ring.

TheOREM 2.3. The elastic energy admits the expansion

$$
a\left(C(R, \varepsilon) ; u_{\varepsilon}, u_{\varepsilon}\right)=a(C(R) ; u, u)+\pi \varepsilon^{2} \mathbb{P} \sigma(u(\widehat{x})) \cdot \nabla^{s} u(\widehat{x})+\mathcal{R}(\varepsilon),
$$

where $\mathbb{P}$ is the Pólya-Szegö polarization tensor [2] and the remainder is uniformly bounded by

$$
|\mathcal{R}(\varepsilon)| \leq \varepsilon^{4} C\|\varphi\|_{H^{1 / 2}\left(\Gamma_{R}\right)} .
$$

Proof. The proof is left to Appendix A. 
Remark 2.4. If the nonhomogeneous Dirichlet boundary condition $\varphi$ satisfies

$$
\|\varphi\|_{H^{1 / 2}\left(\Gamma_{R}\right)} \leq \Lambda_{0}
$$

where $\Lambda_{0}$ is a positive constant, then, in terms of the Fourier coefficients $U_{i}$ of $\varphi$, we have

$$
\sum_{k=-\infty}^{k=+\infty} \sqrt{1+k^{2}}\left|U_{k}\right|^{2} \leq \Lambda_{0} .
$$

The result on asymptotics of the elastic energy is well known; we need to show the estimate for the remainder. The result for the elastic energy implies the asymptotic expansion of the Dirichlet-to-Neumann operators.

Corollary 2.5. The elastic energy concentrated in the ring $C(R, \varepsilon)=B_{R} \backslash \overline{B_{\varepsilon}}$ splits into the energy of the unperturbed disk, the first correction term of order $\varepsilon^{2}$, and the remainder, which is uniformly of the order $\Lambda_{0} \varepsilon^{4}$. This means that the associated Steklov-Poincaré operator admits the asymptotic expansion

$$
\mathcal{A}_{\varepsilon}=\mathcal{A}+f(\varepsilon) \mathcal{B}+\mathcal{R}_{\varepsilon}
$$

in the operator norm $\mathcal{L}\left(H^{1 / 2}\left(\Gamma_{R}\right) ; H^{-1 / 2}\left(\Gamma_{R}\right)\right)$, where $f(\varepsilon)=\varepsilon^{2}, \mathcal{B}$ is a bounded linear operator, and $\left\|\mathcal{R}_{\varepsilon}\right\|_{\mathcal{L}\left(H^{1 / 2}\left(\Gamma_{R}\right) ; H^{-1 / 2}\left(\Gamma_{R}\right)\right)}=o(f(\varepsilon))$.

2.3. Regular domain perturbations. Now, the domain $B_{R}$ is perturbed by an elastic inclusion $\omega_{\varepsilon}$. It means that the elastic constants are different in two subdomains $\omega_{\varepsilon}$ and $B_{R} \backslash \overline{\omega_{\varepsilon}}$ of $B_{R}$. There are three possibilities for $\omega_{\varepsilon}$ in the framework of linear elasticity; i.e., $\omega_{\varepsilon}$ can be considered as an elastic inclusion, but there is also a cavity or a rigid inclusion, which can be considered as the limit cases for the elastic moduli with respect to the so-called contrast parameter; see the monograph [27] for details. We are interested in the asymptotics of the solution of the elasticity problem in the perturbed domain, denoted by $B_{R}$, with respect to the small parameter $\varepsilon \rightarrow 0$. Namely, we want to determine the asymptotics of the energy functional for the auxiliary nonhomogeneous Dirichlet boundary value problem in the domain with a small inclusion. The expansions of the energy furnish the asymptotics of the SteklovPoincaré pseudodifferential operator.

Let $\varepsilon \rightarrow 0$ be a small parameter, and assume that $\omega_{\varepsilon}$, with the characteristic function $x \mapsto \chi_{\varepsilon}(x) \in\{0,1\}$, is a small inclusion of radius $\varepsilon$ and centered at $\widehat{x} \in \Omega$. For the sake of simplicity, we assume that $\omega_{\varepsilon}:=B_{\varepsilon}$. Let us consider the perturbed domain, denoted by the same symbol $B_{R}$, with the variable Hooke's tensor of elastic constants

$$
x \mapsto C_{\varepsilon}(x)=\left(1-\chi_{\varepsilon}(x)\right) C+\gamma \chi_{\varepsilon}(x) C
$$

and with the contrast parameter $\gamma$. In such a setting, the constitutive relation of linear elasticity $\sigma_{\varepsilon}(u)=C_{\varepsilon} \nabla^{s} u$ is now dependent on the small parameter $\varepsilon$. The auxiliary, nonhomogeneous Dirichlet boundary value problem of linear elasticity in $B_{R}$ becomes

$$
u_{\varepsilon}=\varphi \quad \text { on } \Gamma_{R}, \quad u_{\varepsilon} \in H^{1}\left(B_{R}\right): a_{\varepsilon}\left(u_{\varepsilon}, v\right)=0 \quad \forall v \in H_{0}^{1}\left(B_{R}\right),
$$

where

$$
a_{\varepsilon}\left(u_{\varepsilon}, v\right)=\int_{B_{R}} \sigma_{\varepsilon}\left(u_{\varepsilon}\right) \cdot \nabla^{s} v \quad \forall v \in H^{1}\left(B_{R}\right)
$$




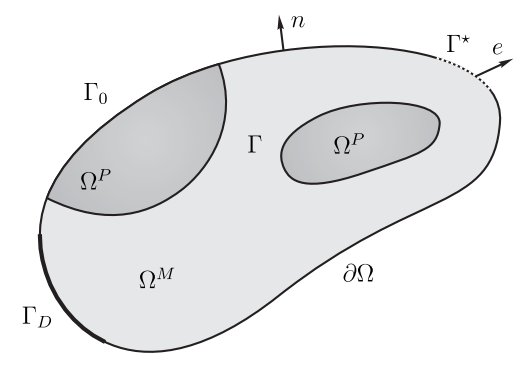

Fig. 2. Piezoelastic coupled problem.

With the solution $u_{\varepsilon}$ of the nonhomogeneous Dirichlet boundary value problem is associated its Neumann trace $T_{\varepsilon}\left(u_{\varepsilon}\right)$ such that the Green's formula is valid,

$$
a_{\varepsilon}\left(u_{\varepsilon}, v\right)+\left(T_{\varepsilon}\left(u_{\varepsilon}\right), v\right)_{\Gamma_{R}}=0 \quad \forall v \in H_{0}^{1}\left(B_{R}\right) .
$$

Thus, the Dirichlet-to-Neumann map depending on the small parameter $\varepsilon$ can be determined in a unique way, in view of the unique solvability of (2.20), from the symmetric, positive bilinear form

$$
H^{1 / 2}\left(\Gamma_{R}\right) \ni \varphi \mapsto\left(\mathcal{A}_{\varepsilon}(\varphi), \varphi\right)_{\Gamma_{R}} \equiv a_{\varepsilon}\left(u_{\varepsilon}, u_{\varepsilon}\right) \in \mathbb{R},
$$

since there are no sources in $B_{R}$. The Dirichlet-to-Neumann map defines the SteklovPoincaré operator for the truncated domain, exterior to $B_{R}$.

Remark 2.6. Following the same procedure as in the previous section, it is possible to show that the asymptotic expansion (2.18) also holds true for the case of the regular perturbations of the disk by an elastic inclusion $\omega_{\varepsilon}$ of the size $\varepsilon \rightarrow 0$, and with the contrast parameter $0<\gamma<\infty$.

3. Problem formulation. Now, we assume that $\Omega=\Omega^{M} \cup \Gamma \cup \Omega^{P}$, where the mutually disjoint open domains $\Omega^{P}$ and $\Omega^{M}$ have the common interface $\Gamma$, as shown in Figure 2. In our notation, $\Omega^{M}$ and $\Omega^{P}$ represent the regions where mechanical and piezoelectric devices, respectively, are located. We consider the coupled model defined in the subdomains $\Omega^{M}$ and $\Omega^{P}$ with different material properties. The models are coupled by the transmission conditions imposed on the interface $\Gamma:=\partial \Omega^{M} \cap \partial \Omega^{P}$. We are looking for a topology of the mechanical part $\Omega^{M}$ that minimizes a given shape functional defined on $\Gamma^{\star} \subset \partial \Omega^{M}$, by keeping the piezoelectric device $\Omega^{P}$ fixed.

In order to define the possible directions of shape-topological modifications in the subdomain $\Omega^{M}$, a small inclusion is introduced. This means that we have at our disposal two materials and try to improve the design by an optimal distribution of more expensive material in the mechanical subdomain. The variation of the shape functional associated with the inclusion $B_{\varepsilon}$ is called the topological derivative [27, 31]. In order to evaluate the topological derivative we need to recover the asymptotics of solutions to the coupled model, find the asymptotics of the shape functional, and introduce the adjoint state in such a way that the result of asymptotic analysis can be used in numerical methods. This approach is used in [26] for the purpose of asymptotic analysis of elasticity boundary value problems in three spatial dimensions.

In this work we propose an alternative approach, which is much simpler compared to the general case considered in [26]. Our goal is the identification of the topological derivative in such a way that it can be directly used in numerical methods. Therefore, 
we combine all the elements of analysis performed in [27,33] and obtain a method which is sufficiently simple to be used in applied shape-topological design. The method is presented for a coupled model of elastic and piezoelectric materials; however, it is general and can be used for more complex models of multiphysics.

3.1. The mechanical model. We are interested in the following system:

$$
\left\{\begin{aligned}
\operatorname{div} \sigma(u)=0 \\
\operatorname{div} S(w, q)=0 \\
\operatorname{div} \phi(w, q)=0
\end{aligned}\right\} \quad \begin{aligned}
& \text { in } \Omega^{M} \\
& \Omega^{P}
\end{aligned}
$$

where the first equation describes the linear elasticity system while the second one gives the coupled system representing the electromechanical interaction phenomenon. The equations are coupled on the interface $\Gamma$. In particular, $\sigma(u)$ is the mechanical stress tensor, $S(w, q)$ is the electromechanical stress tensor, and $\phi(w, q)$ is the electric displacement. The constitutive laws describing the elastic behavior and piezoelectric effects, both in the linearized case of small mechanical deformations and for electric fields, are

$$
\left\{\begin{aligned}
\sigma(u) & =C \nabla^{s} u, \\
S(w, q) & =A \nabla^{s} w+P \nabla q, \\
\phi(w, q) & =P^{T} \nabla^{s} w-K \nabla q,
\end{aligned}\right.
$$

where $u$ and $w$ are the mechanical and electromechanical displacements, respectively, and $q$ is the electric potential. In addition, $C$ and $A$ are the elasticity fourthorder tensors respectively associated to the elastic and electromechanical parts, $P$ the piezoelectric coupling third-order tensor, and $K$ the dielectric second-order tensor. As usual $C, A$, and $K$ satisfy the symmetry conditions $C_{i j k l}=C_{j i k l}=C_{k l i j}$, $A_{i j k l}=A_{j i k l}=A_{k l i j}$, and $K_{i j}=K_{j i}$, whereas $P$ satisfies $P_{i j k}=P_{j i k}$. It is assumed for simplicity that all constitutive tensors are piecewise constant, i.e., constant in each subdomain $\Omega^{M}$ and $\Omega^{P}$. In the case of isotropic elasticity, the tensor $C$ takes the form

$$
C=2 \mu \mathbb{I}+\lambda(I \otimes I),
$$

where $\mu$ and $\lambda$ are the Lamé coefficients and $I$ and $\mathbb{I}$ are the second- and fourth-order identity tensors, respectively. We complement the system (3.1) with the following boundary conditions:

$$
u=0 \text { on } \Gamma_{D}, \quad \sigma(u) n=k u \text { on } \Gamma^{\star}, \quad \text { and } \begin{cases}q=0 & \text { on } \Gamma_{0}, \\ q=\bar{q} & \text { on } \Gamma,\end{cases}
$$

where $\Gamma^{\star}, \Gamma_{D}$, and $\Gamma_{0}$ are parts of the boundary $\partial \Omega$ and $n$ is the outward unit normal vector pointing toward the exterior of $\Omega$. If it is not specified, we consider homogeneous natural (Neumann) boundary conditions of the form $\sigma(u) n=0, S(w, q) n=0$, and $\phi(w, q) \cdot n=0$ on a part of $\partial \Omega$. Finally, we consider the following transmission conditions:

$$
\left\{\begin{aligned}
u & =w \\
\sigma(u) n & =S(w, q) n
\end{aligned} \text { on } \Gamma,\right.
$$

where $n$ is the unit normal vector pointing toward the exterior of $\Omega^{M}$. The variational formulation of the above coupled system reads as follows. 
Problem 3.1. Find $\mathfrak{u} \in \mathcal{V}$ and $q \in \mathcal{Q}$ such that

$$
\left\{\begin{aligned}
\int_{\Omega^{M}} \sigma(u) \cdot \nabla^{s} \eta-\int_{\Gamma^{\star}} k u \cdot \eta+\int_{\Omega^{P}} S(w, q) \cdot \nabla^{s} \eta=0 & \forall \eta \in \mathcal{V}, \\
\int_{\Omega^{P}} \phi(w, q) \cdot \nabla \xi=0 & \forall \xi \in \mathcal{Q}_{0},
\end{aligned}\right.
$$

where $\mathfrak{u}=u$ in $\Omega^{M}$ and $\mathfrak{u}=w$ in $\Omega^{P}$. The space $\mathcal{V}$ of displacements fields is defined as

$$
\mathcal{V}=\left\{v \in H^{1}(\Omega):\left.v\right|_{\Gamma_{D}}=0\right\},
$$

while the electric potential sets $\mathcal{Q}$ and $\mathcal{Q}_{0}$ are respectively defined as

$$
\mathcal{Q}=\left\{q \in H^{1}\left(\Omega^{P}\right):\left.q\right|_{\Gamma}=\bar{q},\left.q\right|_{\Gamma_{0}}=0\right\}, \quad \mathcal{Q}_{0}=\left\{q \in H^{1}\left(\Omega^{P}\right):\left.q\right|_{\partial \Omega^{P}}=0\right\} .
$$

3.2. The shape functional. We are interested in the topology design of piezoelectric actuators, which consist of multiflexible structures actuated by piezoceramic devices that generate an output displacement in a specified direction on the boundary of the actuated part. Therefore, let us introduce a tracking-type shape functional of the form

$$
J(u)=-\int_{\Gamma^{\star}} u \cdot e,
$$

where $e$ is used to denote a given direction on the boundary $\Gamma^{\star}$ (see Figure 2).

3.3. The adjoint state. As mentioned before, we are going to evaluate the shape gradient of functional (3.9). For further simplification, we introduce an adjoint system of the form

$$
\left\{\begin{aligned}
\operatorname{div} \sigma\left(u^{a}\right)=0 \\
\operatorname{div} S^{a}\left(w^{a}, q^{a}\right)=0 \\
\operatorname{div} \phi^{a}\left(w^{a}, q^{a}\right)=0
\end{aligned}\right\} \quad \begin{array}{ll}
\text { in } \Omega^{M}, \\
\Omega^{P},
\end{array}
$$

where $\sigma\left(u^{a}\right), S^{a}\left(w^{a}, q^{a}\right)$, and $\phi^{a}\left(w^{a}, q^{a}\right)$ are, respectively, the adjoint mechanical stress tensor, electromechanical stress tensor, and electrical displacement, given by

$$
\left\{\begin{aligned}
\sigma\left(u^{a}\right) & =C \nabla^{s} u^{a}, \\
S^{a}\left(w^{a}, q^{a}\right) & =A \nabla^{s} w^{a}-P \nabla q^{a}, \\
\phi^{a}\left(w^{a}, q^{a}\right) & =-P^{T} \nabla^{s} w^{a}-K \nabla q^{a},
\end{aligned}\right.
$$

where $u^{a}, w^{a}$, and $q^{a}$ are the adjoint mechanical displacement, electromechanical displacement, and electric potential, respectively. The system (3.10) has the boundary conditions

$$
u^{a}=0 \text { on } \Gamma_{D}, \quad \sigma\left(u^{a}\right) n=k u^{a}+e \text { on } \Gamma^{\star}, \quad \text { and } \quad q^{a}=0 \text { on } \partial \Omega^{P}
$$

and the transmission conditions

$$
\left\{\begin{aligned}
u^{a} & =w^{a} \\
\sigma\left(u^{a}\right) n & =S^{a}\left(w^{a}, q^{a}\right) n
\end{aligned} \quad \text { on } \Gamma .\right.
$$

The variational formulation of the coupled system for adjoint state equations reads as follows. 
Problem 3.2. Find $\mathfrak{u}^{a} \in \mathcal{V}$ and $q^{a} \in \mathcal{Q}_{0}$ such that

$$
\left\{\begin{array}{rlrl}
\int_{\Omega^{M}} \sigma\left(u^{a}\right) \cdot \nabla^{s} \eta-\int_{\Gamma^{\star}} k u^{a} \cdot \eta+\int_{\Omega^{P}} S^{a}\left(w^{a}, q^{a}\right) \cdot \nabla^{s} \eta & =\int_{\Gamma^{\star}} e \cdot \eta & & \forall \eta \in \mathcal{V}, \\
\int_{\Omega^{P}} \phi^{a}\left(w^{a}, q^{a}\right) \cdot \nabla \xi & =0 & \forall \xi \in \mathcal{Q}_{0},
\end{array}\right.
$$

where $\mathfrak{u}^{a}=u^{a}$ in $\Omega^{M}$ and $\mathfrak{u}^{a}=w^{a}$ in $\Omega^{P}$.

4. Topological derivative. The topological derivative of functional (3.9) is evaluated for the insertion of a small inclusion in $\Omega^{M}$ with the material properties depending on the contrast. To describe the topological perturbation of $\Omega^{M}$ we introduce a piecewise constant function $\gamma_{\varepsilon}$ of the form

$$
\gamma_{\varepsilon}=\gamma_{\varepsilon}(x):= \begin{cases}1 & \text { if } x \in \Omega^{M} \backslash \overline{B_{\varepsilon}} \\ \gamma & \text { if } x \in B_{\varepsilon}\end{cases}
$$

where $0<\gamma<\infty$ is the contrast parameter on the material properties and $B_{\varepsilon}(\widehat{x})$ for $\widehat{x} \in \Omega^{M}$. Note that in this case the topologies of the original and perturbed domains are preserved. However, we are introducing a nonsmooth perturbation in the coefficients of the differential operator through the contrast $\gamma_{\varepsilon}$, by changing the material property of the background in a small region $B_{\varepsilon} \subset \Omega^{M}$. Therefore, the sensitivity of the shape functional with respect to the nucleation of an inclusion can also be handle through the topological asymptotic analysis concept, which actually is the best approach for such a problem. The variational formulation associated with the perturbed coupled system reads as follows.

Problem 4.1. Find $\mathfrak{u}_{\varepsilon} \in \mathcal{V}$ and $q_{\varepsilon} \in \mathcal{Q}$ such that

$$
\left\{\begin{aligned}
\int_{\Omega^{M}} \sigma_{\varepsilon}\left(u_{\varepsilon}\right) \cdot \nabla^{s} \eta-\int_{\Gamma^{\star}} k u_{\varepsilon} \cdot \eta+\int_{\Omega^{P}} S\left(w_{\varepsilon}, q_{\varepsilon}\right) \cdot \nabla^{s} \eta=0 & \forall \eta \in \mathcal{V}, \\
\int_{\Omega^{P}} \phi\left(w_{\varepsilon}, q_{\varepsilon}\right) \cdot \nabla \xi=0 & \forall \xi \in \mathcal{Q}_{0},
\end{aligned}\right.
$$

where $\sigma_{\varepsilon}\left(u_{\varepsilon}\right)=\gamma_{\varepsilon} C \nabla^{s} u_{\varepsilon}$. In addition, $\mathfrak{u}_{\varepsilon}=u_{\varepsilon}$ in $\Omega^{M}$ and $\mathfrak{u}_{\varepsilon}=w_{\varepsilon}$ in $\Omega^{P}$.

4.1. Preliminaries. In this paper a coupled model is considered in the domain $\Omega$. The coupled system (3.6) is well-posed and can be written in the strong form as an abstract equation for the unknown functions $U:=(u, w, q)$,

$$
L U=F,
$$

in the appropriate function spaces over the domain $\Omega$, where $F$ is a vector that has the generalized loading system for the problem and $L$ is the matrix of the complete system of equations. The weak form reads

$$
\mathcal{L}(U, \Phi)=(F, \Phi),
$$

with the test functions $\Phi$. The bilinear form associated with the elastic component of the coupled model in the subdomain $\Omega^{M}$ is simply given by standard expression of linear elasticity,

$$
(u, \eta) \mapsto a\left(\Omega^{M} ; u, \eta\right):=\int_{\Omega^{M}} \sigma(u) \cdot \nabla^{s} \eta
$$


in the unperturbed subdomain $\Omega^{M}$, as well as by

$$
(u, \eta) \mapsto a_{\varepsilon}\left(\Omega^{M} ; u, \eta\right):=\int_{\Omega^{M}} \sigma_{\varepsilon}(u) \cdot \nabla^{s} \eta
$$

in the perturbed subdomain by an inclusion. Here $\varepsilon \rightarrow 0$ is the parameter which governs the size of the topological perturbation. In the latter case, the weak formulation of the coupled model also depends on the small parameter $\varepsilon$ and can be rewritten as

$$
\mathcal{L}_{\varepsilon}\left(U_{\varepsilon}, \Phi\right)=(F, \Phi)
$$

or written in the strong form $L_{\varepsilon} U_{\varepsilon}=F$. The perturbed system is also well-posed for $\varepsilon \in\left[0, \varepsilon_{0}\right)$, with $\varepsilon_{0}>0$; i.e., the inverse operator is uniformly bounded: $\left\|U_{\varepsilon}\right\| \leq C\|F\|$ in appropriate norms. However, in the case of a cavity the associated function spaces are obviously dependent on the small parameter $\varepsilon$. The case of an inclusion is therefore the regular perturbation of the problem in the fixed function spaces setting.

We proceed further with the domain decomposition technique in the subdomain $\Omega^{M}:=B_{R} \cup \Gamma_{R} \cup \Omega_{R}^{M}$, where $B_{R}$ is the ball of radius $R$ which contains the topological perturbation denoted by $B_{\varepsilon}, \Gamma_{R} \equiv \partial B_{R}$ stands for the boundary of $B_{R}$, and the remaining subdomain $\Omega_{R}^{M}:=\Omega^{M} \backslash \overline{B_{R}}$ is far from the singular topological domain perturbation.

Let $u_{\varepsilon}$ denote the solution of coupled equations in the perturbed domain $\Omega$, i.e., including the inclusion $B_{\varepsilon}$. We are going to show that the restriction of $u_{\varepsilon}$ to the truncated domain solves a boundary value problem with the nonlocal boundary conditions on $\Gamma_{R}$ defined by the Steklov-Poincaré operator. Since for all $\varepsilon \in\left[0, \varepsilon_{0}\right)$

$$
a_{\varepsilon}\left(\Omega^{M} ; u_{\varepsilon}, \eta\right)=a\left(\Omega_{R}^{M} ; u_{\varepsilon}, \eta\right)+a_{\varepsilon}\left(B_{R} ; u_{\varepsilon}, \eta\right)
$$

and the last term is equivalent to the Steklov-Poincaré component, then by construction

$$
a_{\varepsilon}\left(B_{R} ; u_{\varepsilon}, \eta\right) \equiv\left(\mathcal{A}_{\varepsilon}\left(u_{\varepsilon}\right), \eta\right)_{\Gamma_{R}} .
$$

Henceforth the bilinear form in the topologically perturbed domain $\Omega^{M}$ can be replaced by the bilinear form in the unperturbed domain $\Omega_{R}^{M}$, however with a nonlocal pseudodifferential operator

$$
a_{\varepsilon}\left(\Omega^{M} ; u_{\varepsilon}, \eta\right)=a\left(\Omega_{R}^{M} ; u_{\varepsilon}, \eta\right)+\left(\mathcal{A}_{\varepsilon}\left(u_{\varepsilon}\right), \eta\right)_{\Gamma_{R}} .
$$

This replacement is in fact crucial for the proofs of topological differentiability for the shape functionals defined for the coupled system. Actually, the asymptotic expansion of the solutions to the coupled model in the truncated domain can easily be deduced from the well-posedness of the model. It means that for the linear model, and the regular perturbations of the differential operator, the asymptotic expansion of the Steklov-Poincaré operator,

$$
\mathcal{A}_{\varepsilon}=\mathcal{A}+f(\varepsilon) \mathcal{A}^{\prime}+\mathcal{R}_{\varepsilon}
$$

with $f(\varepsilon) \sim\left|B_{\varepsilon}\right|$, implies the asymptotic expansion of the solutions to the coupled model in the truncated domain $\Omega_{R}^{M} \cup \Gamma_{R} \cup \Omega^{P}$ of the same form,

$$
U_{\varepsilon}=U+f(\varepsilon) U^{\prime}+\widetilde{U}_{\varepsilon}
$$

where $\widetilde{U}_{\varepsilon}$ is the remainder; namely, $\left\|\widetilde{U}_{\varepsilon}\right\|=o(f(\varepsilon))$ in appropriated norms. 
4.2. Topological asymptotic expansion of the Steklov-Poincaré operator. The proposed method of asymptotic analysis is employed now on the coupled system defined in $\Omega_{R}$. The dependence of the model on the small parameter $\varepsilon \rightarrow 0$ occurs in the nonlocal boundary conditions imposed on $\Gamma_{R}$. The variational form of (4.2) restricted to $\Omega_{R}$ is obtained:

$$
\left\{\begin{aligned}
\int_{\Omega^{M} \backslash \overline{B_{R}}} \sigma_{\varepsilon}\left(u_{\varepsilon}\right) \cdot \nabla^{s} \eta+\int_{\partial B_{R}} \mathcal{A}_{\varepsilon}\left(u_{\varepsilon}\right) \cdot \eta & \\
-\int_{\Gamma^{\star}} k u_{\varepsilon} \cdot \eta+\int_{\Omega^{P}} S\left(w_{\varepsilon}, q_{\varepsilon}\right) \cdot \nabla^{s} \eta=0 & \forall \eta \in \mathcal{V}, \\
\int_{\Omega^{P}} \phi\left(w_{\varepsilon}, q_{\varepsilon}\right) \cdot \nabla \xi=0 & \forall \xi \in \mathcal{Q}_{0},
\end{aligned}\right.
$$

where $B_{R}=B_{R}(\widehat{x})$ is a ball of radius $R$ and center at $\widehat{x} \in \Omega^{M}$.

The Steklov-Poincaré operator on the interior boundary $\partial B_{R}$ of the truncated domain $\Omega_{R}$,

$$
\mathcal{A}_{\varepsilon}: \varphi \in H^{1 / 2}\left(\partial B_{R}\right) \rightarrow \sigma_{\varepsilon}\left(v_{\varepsilon}\right) n \in H^{-1 / 2}\left(\partial B_{R}\right),
$$

by construction coincides with the Dirichlet-to-Neumann map of the linear elasticity on the ball $B_{R}$,

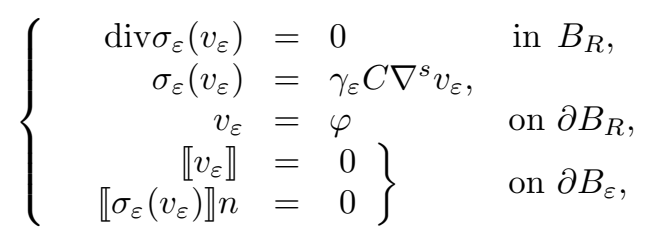

with $\mathcal{A}_{\varepsilon}(\varphi)=\sigma_{\varepsilon}\left(v_{\varepsilon}\right) n$, which assures the identity $v_{\varepsilon}=\left.u_{\varepsilon}\right|_{B_{R}}$ in $B_{R}$, where $u_{\varepsilon}$ is the solution of the perturbed problem in $\Omega$.

Remark 4.2. If the Steklov-Poincaré operator $\varphi \mapsto \mathcal{A}_{\varepsilon}(\varphi)$ of problem (4.13) is the Dirichlet-to-Neumann map defined by (4.15), then the solution to (4.13) coincides with the restriction to $\Omega_{R}$ of the solution to the perturbed problem in $\Omega$.

The identity for the energy functional of (4.15) holds as

$$
\begin{aligned}
0=-\int_{B_{R}} \operatorname{div} \sigma_{\varepsilon}\left(v_{\varepsilon}\right) \cdot v_{\varepsilon} & =\int_{B_{R}} \sigma_{\varepsilon}\left(v_{\varepsilon}\right) \cdot \nabla^{s} v_{\varepsilon}-\int_{\partial B_{R}} \sigma_{\varepsilon}\left(v_{\varepsilon}\right) n \cdot v_{\varepsilon} \\
& =\int_{B_{R}} \sigma_{\varepsilon}\left(v_{\varepsilon}\right) \cdot \nabla^{s} v_{\varepsilon}-\int_{\partial B_{R}} \mathcal{A}_{\varepsilon}(\varphi) \cdot \varphi ;
\end{aligned}
$$

hence the elastic energy in $B_{R}$ equals the energy of the Steklov-Poincaré operator on the boundary. Thus we conclude that the asymptotic expansion of the SteklovPoincaré operator on the common boundary $\partial B_{R}$ equals the asymptotic expansion of the elastic energy in the domain $B_{R}$. Namely,

$$
\int_{B_{R}} \sigma_{\varepsilon}\left(v_{\varepsilon}\right) \cdot \nabla^{s} v_{\varepsilon}=\int_{\partial B_{R}} \mathcal{A}_{\varepsilon}(\varphi) \cdot \varphi
$$

for the mapping defined by (4.15),

$$
\varphi \in H^{1 / 2}\left(\partial B_{R}\right) \rightarrow \sigma_{\varepsilon}\left(v_{\varepsilon}\right) n \in H^{-1 / 2}\left(\partial B_{R}\right) .
$$


Since the operator $\mathcal{A}_{\varepsilon}$ is symmetric, we can also write

$$
\int_{B_{R}} \sigma_{\varepsilon}\left(v_{\varepsilon}\right) \cdot \nabla^{s} v_{\varepsilon}=\left\langle\mathcal{A}_{\varepsilon}(\varphi), \varphi\right\rangle_{\left(H^{-1 / 2} \times H^{1 / 2}\right)\left(\partial B_{R}\right)} .
$$

It is well known that the topological asymptotic expansion for the energy functional takes the following form [27]:

$$
\int_{B_{R}} \sigma_{\varepsilon}\left(v_{\varepsilon}\right) \cdot \nabla^{s} v_{\varepsilon}=\int_{B_{R}} \sigma(v) \cdot \nabla^{s} v+f(\varepsilon) \mathbb{P}_{\gamma} \sigma(v(\widehat{x})) \cdot \nabla^{s} v(\widehat{x})+o(f(\varepsilon)),
$$

where $v=\left.u\right|_{B_{R}}$ is the solution to the original (unperturbed) problem (3.6) and $\mathbb{P}_{\gamma}$ is the Pólya-Szegö polarization tensor [2]. According to the expansion given in Corollary 2.5 and by the symmetry of the Steklov-Poincaré operator, the expansion of the energy functional can also be written as

$$
\left\langle\mathcal{A}_{\varepsilon}(\varphi), \vartheta\right\rangle=\langle\mathcal{A}(\varphi), \vartheta\rangle+f(\varepsilon)\langle\mathcal{B}(\varphi), \vartheta\rangle+\left\langle\mathcal{R}_{\varepsilon}(\varphi), \vartheta\right\rangle,
$$

where $\left\langle\mathcal{R}_{\varepsilon}(\varphi), \vartheta\right\rangle=o(f(\varepsilon))$. Then, from the asymptotic expansion of the energy functional, we get

$$
\langle\mathcal{B}(\varphi), \vartheta\rangle=\mathbb{P}_{\gamma} \sigma(\varphi(\widehat{x})) \cdot \nabla^{s} \vartheta(\widehat{x}) \quad \forall \widehat{x} \in \Omega^{M} .
$$

4.3. Topological asymptotic expansion of the solution. We consider the following ansätze for the solutions $u_{\varepsilon}, w_{\varepsilon}, q_{\varepsilon}$ to the topologically perturbed coupled system (4.2):

$$
\begin{aligned}
u_{\varepsilon} & =u+f(\varepsilon) g+\widetilde{u}_{\varepsilon}, \\
w_{\varepsilon} & =w+f(\varepsilon) h+\widetilde{w}_{\varepsilon}, \\
q_{\varepsilon} & =q+f(\varepsilon) p+\widetilde{q}_{\varepsilon},
\end{aligned}
$$

where $u, w, q$ are solutions to the original (unperturbed) coupled system (3.6); $g, h, p$ are the first-order asymptotic correction terms; and $\widetilde{u}_{\varepsilon}, \widetilde{w}_{\varepsilon}, \widetilde{q}_{\varepsilon}$ are the remainders. Now, we plug these ansätze into (4.13) and collect the terms with the same powers of $\varepsilon$ to obtain three boundary value problems. The first problem for $u, w$, and $q$ is

$$
\left\{\begin{aligned}
\int_{\Omega^{M} \backslash \overline{B_{R}}} \sigma(u) \cdot \nabla^{s} \eta+\int_{\partial B_{R}} \mathcal{A}(u) \cdot \eta & \\
-\int_{\Gamma^{\star}} k u \cdot \eta+\int_{\Omega^{P}} S(w, q) \cdot \nabla^{s} \eta=0 & \forall \eta \in \mathcal{V}, \\
\int_{\Omega^{P}} \phi(w, q) \cdot \nabla \xi=0 & \forall \xi \in \mathcal{Q}_{0} .
\end{aligned}\right.
$$

The second problem for $g, h$, and $p$ is

$$
\left\{\begin{aligned}
\int_{\Omega^{M} \backslash \overline{B_{R}}} \sigma(g) \cdot \nabla^{s} \eta+\int_{\partial B_{R}}(\mathcal{A}(g)+\mathcal{B}(u)) \cdot \eta & \\
-\int_{\Gamma^{*}} k g \cdot \eta+\int_{\Omega^{P}} S(h, p) \cdot \nabla^{s} \eta=0 & \forall \eta \in \mathcal{V}, \\
\int_{\Omega^{P}} \phi(h, p) \cdot \nabla \xi=0 & \forall \xi \in \mathcal{Q}_{0},
\end{aligned}\right.
$$


and the third problem for the remainders $\widetilde{u}_{\varepsilon}, \widetilde{w}_{\varepsilon}$, and $\widetilde{q}_{\varepsilon}$ is

$$
\left\{\begin{array}{rlrl}
\int_{\Omega^{M} \backslash \overline{B_{R}}} \sigma_{\varepsilon}\left(\widetilde{u}_{\varepsilon}\right) \cdot \nabla^{s} \eta+\int_{\partial B_{R}} \mathcal{A}_{\varepsilon}\left(\widetilde{u}_{\varepsilon}\right) \cdot \eta & \\
-\int_{\Gamma^{*}} k \widetilde{u}_{\varepsilon} \cdot \eta+\int_{\Omega^{P}} S\left(\widetilde{w}_{\varepsilon}, \widetilde{q}_{\varepsilon}\right) \cdot \nabla^{s} \eta & =\int_{\partial B_{R}} \widetilde{F}_{\varepsilon} \cdot \eta & & \forall \eta \in \mathcal{V}, \\
\int_{\Omega^{P}} \phi\left(\widetilde{w}_{\varepsilon}, \widetilde{q}_{\varepsilon}\right) \cdot \nabla \xi & =0 & & \forall \xi \in \mathcal{Q}_{0},
\end{array}\right.
$$

where the source $\mathfrak{F}_{\varepsilon}$ is given by

$$
\mathfrak{F}_{\varepsilon}=-\left(\mathcal{R}_{\varepsilon}(u)+f(\varepsilon) \mathcal{R}_{\varepsilon}(g)+f(\varepsilon)^{2} \mathcal{B}(g)\right) .
$$

The estimations $\left\|\widetilde{u}_{\varepsilon}\right\|_{H^{1}\left(\Omega^{M} \backslash \overline{B_{R}}\right)}=o(f(\varepsilon)),\left\|\widetilde{w}_{\varepsilon}\right\|_{H^{1}\left(\Omega^{P}\right)}=o(f(\varepsilon))$, and $\left\|\widetilde{q}_{\varepsilon}\right\|_{H^{1}\left(\Omega^{P}\right)}=$ $o(f(\varepsilon))$ hold true for the remainders [27].

4.4. Topological asymptotic expansion of the shape functional. Now we are in position to establish the asymptotic expansion of the shape functional and obtain its topological derivative. After introducing the first ansatz in the shape functional associated to the perturbed problem, we have

$$
\begin{aligned}
J\left(u_{\varepsilon}\right) & =-\int_{\Gamma^{*}}\left(u+f(\varepsilon) g+\widetilde{u}_{\varepsilon}\right) \cdot e \\
& =-\int_{\Gamma^{\star}} u \cdot e-f(\varepsilon) \int_{\Gamma^{\star}} g \cdot e-\int_{\Gamma^{\star}} \widetilde{u}_{\varepsilon} \cdot e \\
& =J(u)-f(\varepsilon) \int_{\Gamma^{\star}} g \cdot e+o(f(\varepsilon)) .
\end{aligned}
$$

Now, let us rewrite the adjoint system (3.14) as

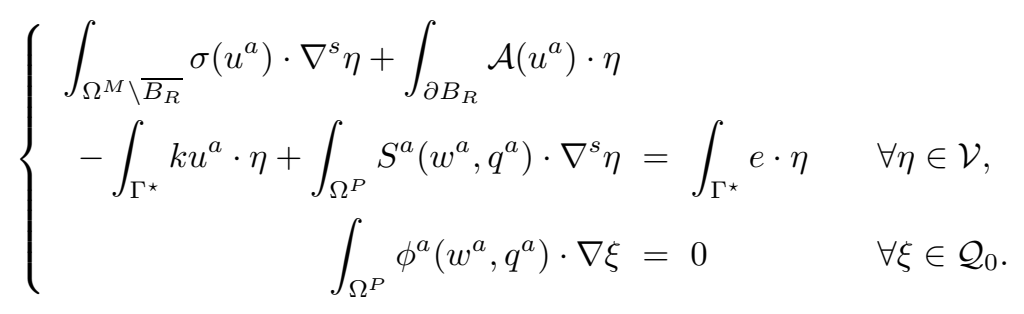

By taking $g, h$, and $p$ as the test functions in (4.31), we have the following equalities:

$$
\begin{aligned}
\int_{\Omega^{M} \backslash \overline{B_{R}}} \sigma\left(u^{a}\right) \cdot \nabla^{s} g+\int_{\partial B_{R}} \mathcal{A}\left(u^{a}\right) \cdot g-\int_{\Gamma^{\star}} k u^{a} \cdot g \\
\quad+\int_{\Omega^{P}} A \nabla^{s} w^{a} \cdot \nabla^{s} h-\int_{\Omega^{P}} P \nabla q^{a} \cdot \nabla^{s} h=\int_{\Gamma^{\star}} e \cdot g \\
-\int_{\Omega^{P}} P^{\top} \nabla^{s} w^{a} \cdot \nabla p-\int_{\Omega^{P}} K \nabla q^{a} \cdot \nabla p=0 .
\end{aligned}
$$

On the other hand, by taking $u^{a}, w^{a}$, and $q^{a}$ as the test functions in (4.27), we obtain

$$
\begin{aligned}
\int_{\Omega^{M} \backslash \overline{B_{R}}} \sigma(g) \cdot \nabla^{s} u^{a}+ & \int_{\partial B_{R}}(\mathcal{A}(g)+\mathcal{B}(u)) \cdot u^{a}-\int_{\Gamma^{\star}} k g \cdot u^{a} \\
+ & \int_{\Omega^{P}} A \nabla^{s} h \cdot \nabla^{s} w^{a}+\int_{\Omega^{P}} P \nabla p \cdot \nabla^{s} w^{a}=0, \\
& \int_{\Omega^{P}} P^{\top} \nabla^{s} h \cdot \nabla q^{a}-\int_{\Omega^{P}} K \nabla p \cdot \nabla q^{a}=0 .
\end{aligned}
$$


Combining the above equalities yields the following important result:

$$
\begin{aligned}
\int_{\Gamma^{\star}} e \cdot g & =-\int_{\partial B_{R}} \mathcal{B}(u) \cdot u^{a} \\
& =-\left\langle\mathcal{B}(u), u^{a}\right\rangle_{\left(H^{-1 / 2} \times H^{1 / 2}\right)\left(\partial B_{R}\right)} \\
& =-\mathbb{P}_{\gamma} \sigma(u(\widehat{x})) \cdot \nabla^{s} u^{a}(\widehat{x}),
\end{aligned}
$$

where we have considered the symmetry of the bilinear forms. Finally, the topological asymptotic expansion of the shape functional leads to

$$
J\left(u_{\varepsilon}\right)=J(u)+f(\varepsilon) \mathbb{P}_{\gamma} \sigma(u(\widehat{x})) \cdot \nabla^{s} u^{a}(\widehat{x})+o(f(\varepsilon)) .
$$

By assuming that the inclusion is far from the piezoelectric part, the topological derivative is given by the following closed formula:

$$
\mathcal{T}_{\gamma}(\widehat{x})=\mathbb{P}_{\gamma} \sigma(u(\widehat{x})) \cdot \nabla^{s} u^{a}(\widehat{x}) \quad \forall \widehat{x} \in \Omega^{M},
$$

where $u$ and $u^{a}$ are solutions to the original unperturbed direct (i.e., (3.6)) and adjoint (i.e., (3.14)) systems, respectively.

Corollary 4.3. In two spatial dimensions, the function $f(\varepsilon)=\varepsilon^{2}$ and the polarization tensor for inclusions $(0<\gamma<\infty)$ reads [27] (see also [10])

$$
\mathbb{P}_{\gamma}=\pi \frac{1-\gamma}{1+\gamma \beta}\left((1+\beta) \mathbb{I}+\frac{1}{2}(\alpha-\beta) \frac{1-\gamma}{1+\gamma \alpha} I \otimes I\right),
$$

with the constants $\alpha$ and $\beta$ given by

$$
\alpha=\frac{\lambda+\mu}{\mu} \quad \text { and } \quad \beta=\frac{\lambda+3 \mu}{\lambda+\mu} .
$$

In three spatial dimensions, the function $f(\varepsilon)=\varepsilon^{3}$ and the polarization tensor for holes $(\gamma=0)$ yield [27] (see also [12])

$$
\mathbb{P}_{0}=2 \pi \frac{1-\nu}{7-5 \nu}\left(10 \mathbb{I}-\frac{1-5 \nu}{1-2 \nu} I \otimes I\right),
$$

where $\nu$ is the Poisson ratio.

5. Numerical implementation. A numerical procedure has been defined to evaluate the topological derivative $\mathcal{T}_{\gamma}(\widehat{x})$ of the shape functional $J(u)$ in (3.9).

First, the constitutive properties for the direct problem (3.1) are defined according to (3.2). Thus, the tensor $C$ is defined for the elastic part $\Omega^{M}$, and tensors $A, P$, and $K$ are defined for the electromechanical part $\Omega^{P}$. The boundary conditions for the direct problem (3.1) are also specified according to (3.4). Thus, the mechanical displacement is specified as $u=0$ on $\Gamma_{D}, \sigma(u) n-k u=0$ on $\Gamma^{\star}$ (where $k$ is the stiffness of the spring), and the electric potential $q$ is defined on $\partial \Omega^{P}=\Gamma \cup \Gamma_{0}$. Next, either problem (3.1) or (3.6) is solved to obtain the displacement field $u$ in $\Omega^{M}$.

Then, we move to the adjoint problem, by first defining the constitutive properties for problem (3.10) according to (3.11). The tensors $C, A, P$, and $K$ are the same; however, in (3.11) there is a change in the sign of $P$. The boundary conditions for the adjoint problem (3.10) are also defined according to (3.12). Thus, the adjoint mechanical displacement is specified as $u^{a}=0$ on $\Gamma_{D}, \sigma\left(u^{a}\right) n-k u^{a}=e$ on $\Gamma^{\star}$ 
(where $k$ is the stiffness of the spring and $e$ is the prescribed direction (vector) for the maximization of the displacement $u$ according to (3.9)), and the adjoint electric potential is defined as $q^{a}=0$ on $\partial \Omega^{P}$. Next, either problem (3.10) or (3.14) is solved to obtain the adjoint displacement field $u^{a}$ in $\Omega^{M}$. Thus, the adjoint problem has the same structure of the direct problem (3.1), with a change in the sign of the tensor $P$ and homogeneous boundary conditions on $\Gamma_{D}$ and $\partial \Omega^{P}$, and nonhomogeneous Robin boundary condition on $\Gamma^{\star}$.

With the direct and adjoint displacements, $u$ and $u^{a}$ in $\Omega^{M}$, and the values of $\gamma$, the topological derivative $\mathcal{T}_{\gamma}(\widehat{x})$ of the shape functional $J(u)$ in $(3.9)$ is calculated at each point $\widehat{x} \in \Omega^{M}$ by using (4.38).

Remark 5.1. For the topological derivative evaluation, we need only the stress associated to the direct displacement $u$ and the strain associated to the adjoint displacement $u^{a}$ in the mechanical part of the domain $\Omega^{M}$. In addition, the Poisson ratio $\nu$ is used to calculate the constants $\alpha$ and $\beta$ in (4.40).

In order to simplify the numerical implementation we consider that the elastic body $\Omega^{M}$ is decomposed into two subdomains $\Omega^{s}$ and $\Omega^{w}$. The domain $\Omega^{s}=\Omega^{M} \backslash \overline{\Omega^{w}}$ represents the elastic part, while $\Omega^{w} \subset \Omega^{M}$ is filled with a very complacent material, used to mimic voids. This procedure allows us to work in a fixed computational domain. Now, we introduce a volume constraint in $\Omega^{s}$ of the form $\left|\Omega^{s}\right| \leq M$. Therefore, the optimization problem we are dealing with can be written as follows:

$$
\underset{\Omega^{s} \subset \Omega^{M}}{\operatorname{Minimize}} J_{\rho}(u)=J(u)+\rho\left|\Omega^{s}\right|,
$$

where $\rho$ is a fixed multiplier used to target the volume $M$.

Having made the above considerations, the topological derivative-based optimization algorithm devised in [3] stands out as a particularly well-suited choice for solving the optimization problem that we are dealing with. The procedure relies on a levelset domain representation [28] and the approximation of the topological optimality conditions by a fixed point iteration. In particular, the algorithm displays a marked ability to produce general topological domain changes uncommon to other methodologies based on a level-set representation, and it has been successfully applied in [3] to topology optimization in the context of two dimensional elasticity and flow through porous media. For completeness, the algorithm is outlined in the following. For further details we refer the reader to [3].

With the adoption of a level-set domain representation, the strong material is characterized by a function $\Psi \in L^{2}\left(\Omega^{M}\right)$ such that

$$
\Omega^{s}=\left\{x \in \Omega^{M}: \Psi(x)<0\right\},
$$

whereas the weak material domain is defined by

$$
\Omega^{w}=\left\{x \in \Omega^{M}: \Psi(x)>0\right\} .
$$

Now, let us consider the topological derivative $\mathcal{T}_{\gamma}^{\rho}(\widehat{x})$ of the shape functional $J_{\rho}(u)$ in (5.1). According to [3], an obvious sufficient condition of local optimality for an optimization problem for the class of perturbations consisting of circular inclusions is

$$
\mathcal{T}_{\gamma}^{\rho}(\widehat{x})>0 \quad \forall \widehat{x} \in \Omega^{M} .
$$

To devise a level-set-based algorithm whose aim is to produce a topology that satisfies (5.4), it is convenient to define the function

$$
G(\widehat{x})= \begin{cases}-G_{s}(\widehat{x}) & \text { if } \widehat{x} \in \Omega^{s} \\ +G_{w}(\widehat{x}) & \text { if } \widehat{x} \in \Omega^{w}\end{cases}
$$


with $G_{s}(\widehat{x})$ and $G_{w}(\widehat{x})$ given by

$$
\begin{array}{rllll}
G_{s}(\widehat{x}):=\mathcal{T}_{\gamma}^{\rho}(\widehat{x}) & \text { with } & \gamma=\gamma^{*} & \text { and } & \widehat{x} \in \Omega^{s}, \\
G_{w}(\widehat{x}):=\mathcal{T}_{\gamma}^{\rho}(\widehat{x}) & \text { with } & \gamma=\frac{1}{\gamma^{*}} & \text { and } & \widehat{x} \in \Omega^{w},
\end{array}
$$

where $0<\gamma^{*} \ll 1$ is the material parameter threshold used to mimic voids.

With the above definitions and (5.2)-(5.3) it can be easily established that the sufficient condition (5.4) is satisfied if the following equivalence relation between $g$ and the level-set function $\Psi$ holds:

$$
\exists \tau>0 \quad \text { s.t. } G=\tau \Psi,
$$

or, equivalently,

$$
\theta:=\arccos \left[\frac{\langle G, \Psi\rangle_{L^{2}\left(\Omega^{M}\right)}}{\|G\|_{L^{2}\left(\Omega^{M}\right)}\|\Psi\|_{L^{2}\left(\Omega^{M}\right)}}\right]=0
$$

where $\theta$ is the angle between the vectors $G$ and $\Psi$ in $L^{2}\left(\Omega^{M}\right)$.

Let us now explain the algorithm. We start by choosing an initial level-set function $\Psi_{0} \in L^{2}\left(\Omega^{M}\right)$. In a generic iteration $n$, we compute function $G_{n}$ associated with the level-set function $\Psi_{n} \in L^{2}\left(\Omega^{M}\right)$. Thus, the new level-set function $\Psi_{n+1}$ is updated according to the following linear combination between the functions $G_{n}$ and $\Psi_{n}$ :

$$
\begin{gathered}
\Psi_{0} \in L^{2}\left(\Omega^{M}\right), \\
\Psi_{n+1}=\frac{1}{\sin \theta_{n}}\left[\sin \left((1-\kappa) \theta_{n}\right) \Psi_{n}+\sin \left(\kappa \theta_{n}\right) \frac{G_{n}}{\left\|G_{n}\right\|_{L^{2}\left(\Omega^{M}\right)}}\right] \quad \forall n \in \mathbb{N},
\end{gathered}
$$

where $\theta_{n}$ is the angle between $G_{n}$ and $\Psi_{n}$, and $\kappa$ is a step size determined by a linearsearch performed in order to decrease the value of the objective function $J_{\rho}\left(u_{n}\right)$, with $u_{n}$ solution to (3.1) associated with $\Psi_{n}$. The process ends when the condition $\theta_{n} \leq \epsilon_{\theta}$ is satisfied in some iteration, where $\epsilon_{\theta}$ is a given small numerical tolerance. In particular, we can choose

$$
\Psi_{0} \in \mathcal{S}=\left\{\varphi \in L^{2}\left(\Omega^{M}\right):\|\varphi\|_{L^{2}\left(\Omega^{M}\right)}=1\right\},
$$

and by construction, $\Psi_{n+1} \in \mathcal{S} \forall n \in \mathbb{N}$. If at some iteration $n$ the linear-search step size $\kappa$ is found to be smaller than a given numerical tolerance $\epsilon_{\kappa}>0$ and the optimality condition is not satisfied, namely $\theta_{n}>\epsilon_{\theta}$, then a uniform mesh refinement of the hold-all domain $\Omega$ is carried out and the iterative process is continued.

TABLE 1

PZT-5A properties.

\begin{tabular}{ccccccccccc}
\hline$\varrho$ & $c_{11}$ & $c_{12}$ & $c_{13}$ & $c_{33}$ & $c_{44}$ & $e_{13}$ & $e_{15}$ & $e_{33}$ & $\varepsilon_{11}$ & $\varepsilon_{33}$ \\
\hline 6.080 & 137.0 & 69.7 & 71.6 & 124.0 & 31.4 & -4.0 & 10.4 & 13.8 & 7.9473 & 5.1507 \\
$\mathrm{~g} / \mathrm{cm}^{3}$ & $\mathrm{GPa}$ & $\mathrm{GPa}$ & $\mathrm{GPa}$ & $\mathrm{GPa}$ & $\mathrm{GPa}$ & $\mathrm{C} / \mathrm{m}^{2}$ & $\mathrm{C} / \mathrm{m}^{2}$ & $\mathrm{C} / \mathrm{m}^{2}$ & $\mathrm{nF} / \mathrm{m}$ & $\mathrm{nF} / \mathrm{m}$ \\
\hline
\end{tabular}

6. Numerical experiments. Several implementation examples are presented in this section. In all of them, the materials considered are PZT-5A in fixed piezoelectric domains and nickel or titanium in optimizable metallic domains; the properties of these materials are given in Tables 1 and 2. Finally, the algorithmic parameters are set as $\gamma^{*}=1 \times 10^{-3}, \epsilon_{\theta}=1^{o}$, and $\epsilon_{\kappa}=1 \times 10^{-4}$, while $\rho$ is targeted according to the required final volume $M$. 
TABLE 2

Nickel and titanium properties.

\begin{tabular}{crl}
\hline & Nickel & Titanium \\
\hline$\varrho\left(\mathrm{g} / \mathrm{cm}^{3}\right)$ & 8.908 & 4.507 \\
$E(\mathrm{GPa})$ & 204.6763 & 109.4027 \\
$\nu$ & 0.2866 & 0.2866 \\
\hline
\end{tabular}

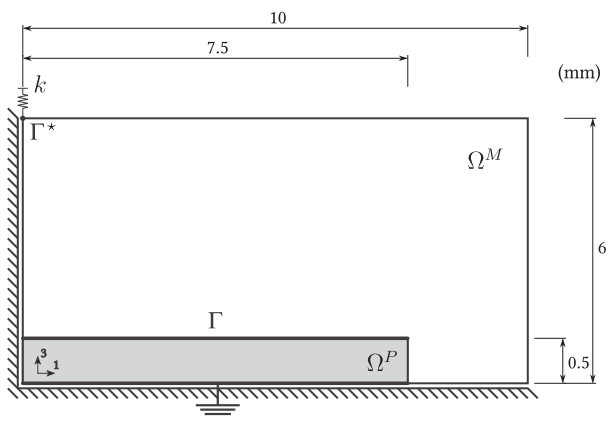

FIG. 3. Example 1: Design domain for piezoelectric moonie (dimensions in $\mathrm{mm}$ ).

6.1. Example 1: Moonie. The first implementation example is the optimization of a moonie, which is a widespread device used to amplify the displacements generated by piezoelectric ceramics. In particular, the design domain considered is shown in Figure 3, in which only one quadrant of the complete domain is represented, based on horizontal and vertical symmetry assumptions. The objective is the maximization of the outward output displacement in the region $\Gamma^{\star}$ in response to some electric potential imposed on the electrode $\Gamma$, from the fixed ceramic domain denoted by $\Omega^{P}$. In the output region $\Gamma^{\star}$, a spring $k$ ensures enough stiffness to the resulting topology obtained within the metallic design domain $\Omega^{s}$.

In the following results, the domain is discretized with a regular mesh of 1920 linear triangle elements at the beginning of the optimization procedure, and 491520 elements at the end. The electric potential applied to $\Gamma$ is $-100 \mathrm{~V}$.

In Figure 4, the results for two different volume fractions $V F$ are shown for both nickel and titanium design domains, with the spring stiffness set to $1 \mathrm{kN} / \mathrm{mm}$. The deformed configuration of a selected result is shown in Figure 5. Furthermore, Figure 6 depicts the impact that the variation of the spring stiffness $k$ has on the final topology obtained, targeting the same final volume. Also in this case, the deformed configuration of a selected result is presented in Figure 7. As the stiffness value is increased, the coupling structure becomes stiffer and the hinges disappear. The output displacements of each resulting topology presented are listed in Table 3.

The method gives a quite clear topology. We notice the presence of flexible hinges in the design, which also appear for the results obtained by using topology optimization based on the density method [29]. From the deformed configurations we notice that the actuator generates the desired displacement.

6.2. Example 2: Inverter. The second example considers the same domain from the previous section; however, the output displacement region $\Gamma^{\star}$ is changed, as depicted in Figure 8. This apparently simple modification in the design domain actually implies a completely different mechanism, since it seeks an output displacement contrary to the natural movement of the structure, and this is the reason it is called an inverter. The design domain remains $\Omega^{s}$, the objective is still the maximization of 


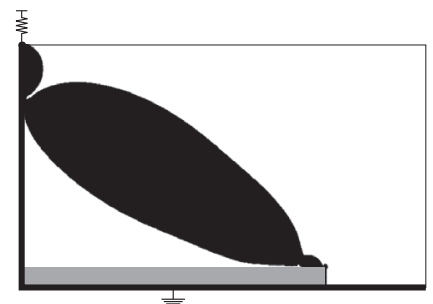

(a) $V F=0.34$

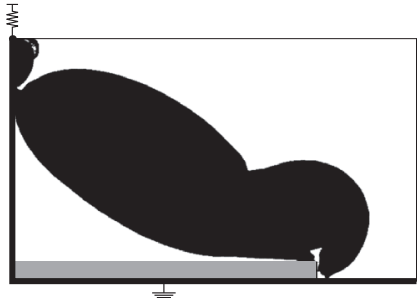

(c) $V F=0.46$.

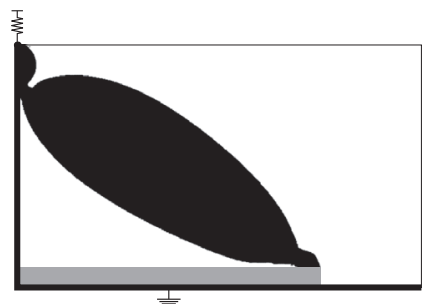

(b) $V F=0.34$.

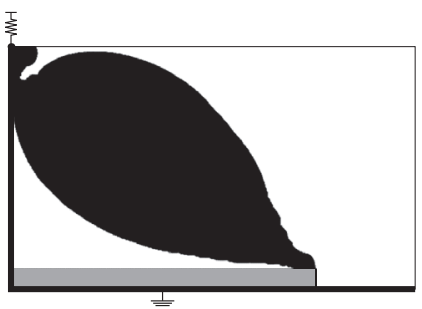

(d) $V F=0.46$.

FIG. 4. Example 1: Results for nickel (left) and titanium (right) for different volume constraints $V F$.

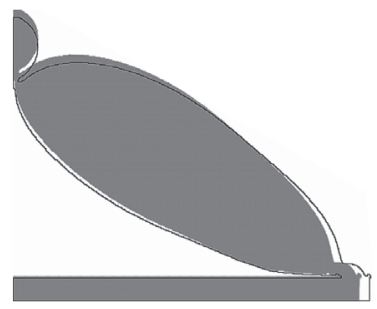

FIG. 5. Example 1: Deformed configuration for result from Figure 4(a).

TABLE 3

Example 1: Output displacements of moonie resulting topologies.

\begin{tabular}{lrr}
\hline & Volume fraction & Displacement $(\mu \mathrm{m})$ \\
\hline 4(a) Nickel $1 \mathrm{kN} / \mathrm{mm}$ & 0.34 & 144.58 \\
4(c) Nickel $1 \mathrm{kN} / \mathrm{mm}$ & 0.46 & 148.77 \\
\hline 6(a) Nickel $1 \mathrm{kN} / \mathrm{mm}$ & 0.30 & 138.59 \\
6(c) Nickel $10 \mathrm{kN} / \mathrm{mm}$ & 0.30 & 39.13 \\
\hline 4(b) Titanium $1 \mathrm{kN} / \mathrm{mm}$ & 0.34 & 133.49 \\
4(d) Titanium $1 \mathrm{kN} / \mathrm{mm}$ & 0.46 & 133.93 \\
\hline 6(b) Titanium $1 \mathrm{kN} / \mathrm{mm}$ & 0.30 & 130.06 \\
6(d) Titanium $10 \mathrm{kN} / \mathrm{mm}$ & 0.30 & 33.92 \\
\hline
\end{tabular}

the outward output displacement in $\Gamma^{\star}$, and all symmetry assumptions are also valid.

The domain is again discretized with the same mesh as in the previous example; the electric potential applied to $\Gamma$ is $-100 \mathrm{~V}$, and the spring stiffness is set to $1 \mathrm{kN} / \mathrm{mm}$. Figure 9 shows the results for two different volume constraints by considering nickel and titanium design domains. The deformed configuration of a selected result is shown in Figure 10. The output displacements of each resulting topology presented are listed in Table 4. 


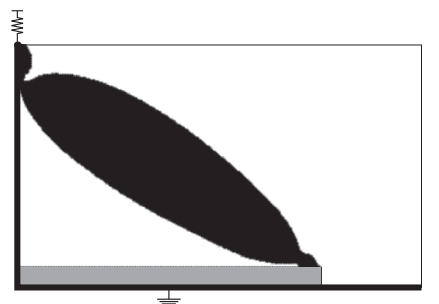

(a) $k=1 \mathrm{kN} / \mathrm{mm}$.

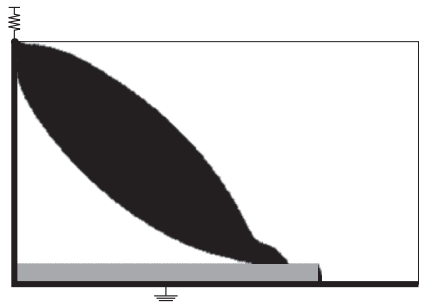

(c) $k=10 \mathrm{kN} / \mathrm{mm}$.

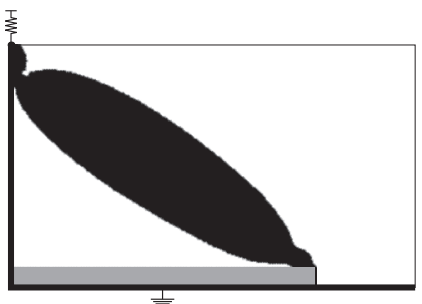

(b) $k=1 \mathrm{kN} / \mathrm{mm}$

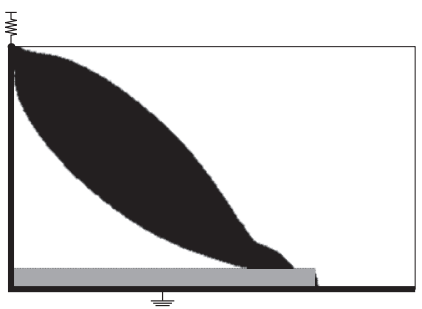

(d) $k=10 \mathrm{kN} / \mathrm{mm}$.

FIG. 6. Example 1. Results for nickel (left) and titanium (right) for $V F=0.30$ and different spring stiffness $k$.

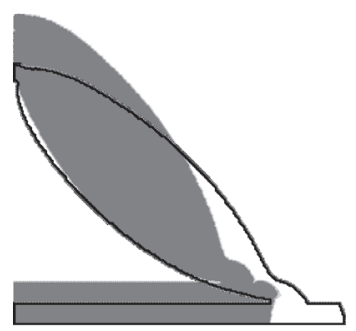

FIG. 7. Example 1: Deformed configuration for result from Figure 6(d).

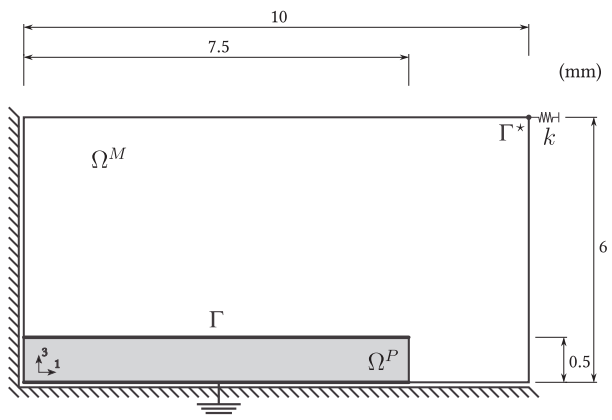

FIG. 8. Example 2: Design domain for piezoelectric inverter (dimensions in mm).

The presence of flexible hinges is also noticed in the results. Further, results from Figures 9(a) and 9(b) show that the method is clearly able to obtain the topology and not only the external shape of the coupling structure. From the deformed configuration we noticed that the inverter generates a displacement opposite to its natural behavior. 


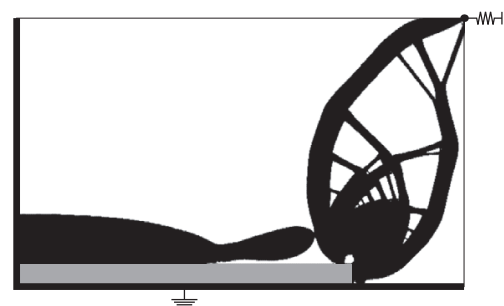

(a) $V F=0.30$

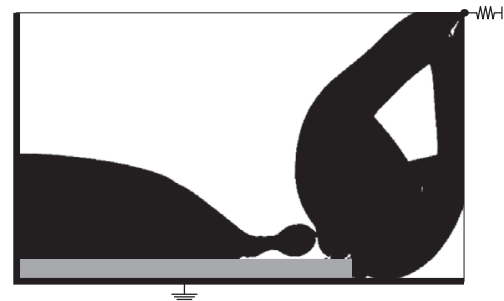

(c) $V F=0.50$

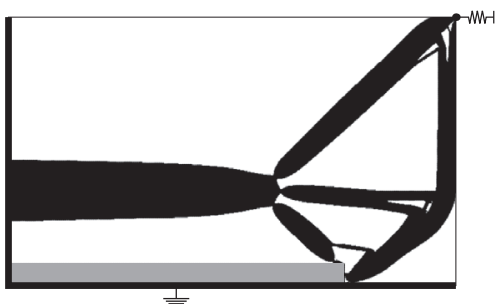

(b) $V F=0.30$.

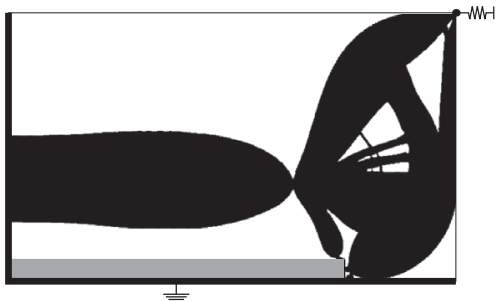

(d) $V F=0.50$.

FIG. 9. Example 2: Results for nickel (left) and titanium (right) for different volume constraints $V F$.

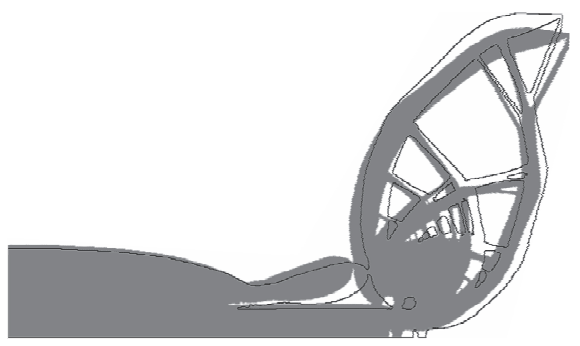

FIG. 10. Example 2: Deformed configuration for result from Figure 9(a).

TABLE 4

Example 2: Output displacements of inverter resulting topologies.

\begin{tabular}{lrr}
\hline & Volume fraction & Displacement $(\mu \mathrm{m})$ \\
\hline 9(a) Nickel $1 \mathrm{kN} / \mathrm{mm}$ & 0.30 & 71.99 \\
9(c) Nickel $1 \mathrm{kN} / \mathrm{mm}$ & 0.50 & 70.37 \\
\hline 9(b) Titanium $1 \mathrm{kN} / \mathrm{mm}$ & 0.30 & 104.88 \\
9(d) Titanium $1 \mathrm{kN} / \mathrm{mm}$ & 0.50 & 112.55 \\
\hline
\end{tabular}

6.3. Example 3: Gripper. The last implementation example investigates the optimization of a gripper. In this case, a half part of the complete device is considered, with horizontal symmetry, as indicated in Figure 11. Unlike the previous examples, the desired output displacement direction is inwards, since a normally closed gripper is being considered. Given the characteristic of the $\Gamma^{\star}$ region, $k$ is a distributed spring.

In the following results, the domain is discretized with a regular mesh of 1596 linear triangle elements at the beginning of the optimization procedure, and 408576 elements at the end. The electric potential applied to $\Gamma$ is $-100 \mathrm{~V}$, and the spring stiffness is set to $1 \mathrm{kN} / \mathrm{mm}$. Figure 12 shows the results for two different volume 


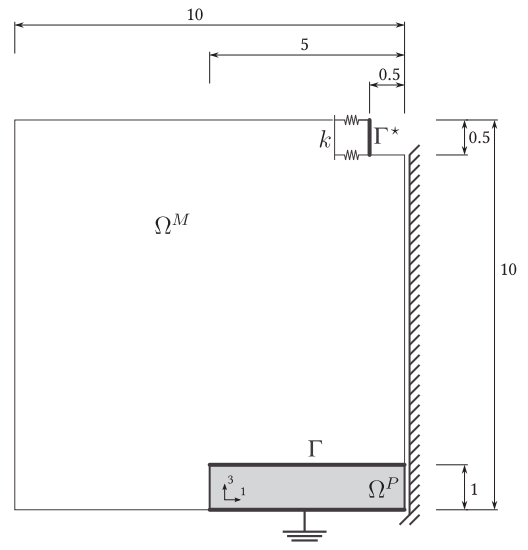

FIG. 11. Example 3: Design domain for piezoelectric gripper (dimensions in $\mathrm{mm}$ ).

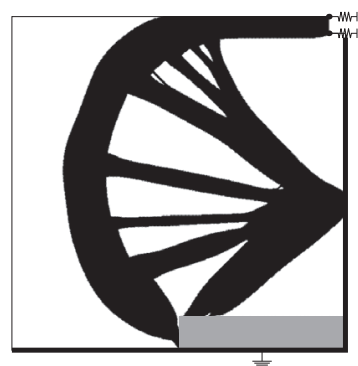

(a) $V F=0.40$.

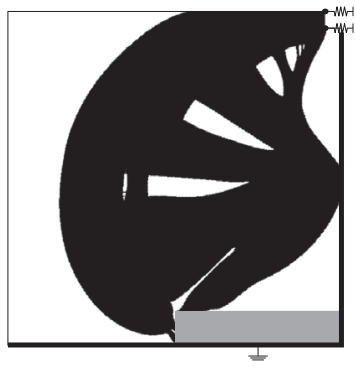

(c) $V F=0.60$.

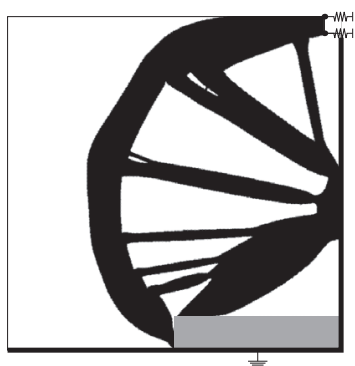

(b) $V F=0.40$

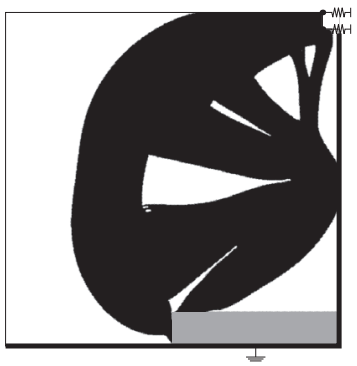

(d) $V F=0.60$.

FIG. 12. Example 3: Results for nickel (left) and titanium (right) for different volume constraints $V F$.

constraints by considering nickel and titanium design domains. The deformed configuration of a selected result is shown in Figure 13. The output displacements of each resulting topology presented are listed in Table 5 .

Again, results from Figure 12 show that the method is able to obtain a clear topology. From the deformed configurations we notice that the gripper generates the desired movement.

7. Conclusions. In this paper the topological derivatives of the tracking-type shape functional for the coupled models of elasto-piezoelectric type are derived in two and three spatial dimensions. The associated shape optimization problems are 


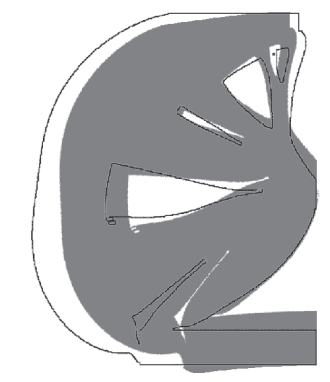

FIG. 13. Example 3: Deformed configuration for result from Figure 12(d).

TABLE 5

Example 3: Output displacements of gripper resulting topologies.

\begin{tabular}{lrr}
\hline & Volume fraction & Displacement $(\mu \mathrm{m})$ \\
\hline $12($ a) Nickel $1 \mathrm{kN} / \mathrm{mm}$ & 0.40 & -17.81 \\
$12($ c) Nickel $1 \mathrm{kN} / \mathrm{mm}$ & 0.60 & -21.51 \\
\hline $12(\mathrm{~b})$ Titanium $1 \mathrm{kN} / \mathrm{mm}$ & 0.40 & -14.76 \\
12 (d) Titanium $1 \mathrm{kN} / \mathrm{mm}$ & 0.60 & -17.10 \\
\hline
\end{tabular}

already analyzed from the point of view of shape optimization in previous work. In this paper the preceding results are completed by the topological asymptotic analysis. The remarkable simplicity of the closed form sensitivity given by (4.38) is to be noted. In fact, once the solutions $u, w, q$ and $u^{a}, w^{a}, q^{a}$ to the original (unperturbed) direct (3.6) and adjoint (3.14) coupled systems have been obtained, the topological derivative $\mathcal{T}_{\gamma}(\widehat{x})$ can be evaluated for all $\widehat{x} \in \Omega^{M}$. The information provided by $\mathcal{T}_{\gamma}(\widehat{x})$ can potentially be used in a number of practical applications such as, for example, the shape-topological design of microtools. In particular, some numerical experiments of topology optimization of piezoelectric actuators have been presented.

Appendix A. Proof of Theorem 2.3. Let us consider the plane elasticity boundary value problem in a ring $C(R, \varepsilon)$. In order to establish the exact formula for the Steklov-Poincaré operator on $\Gamma_{R}$, we use the analytic form of the solution for the elasticity system in the ring, with the nonhomogeneous Dirichlet displacement condition on the outer boundary $\Gamma_{R}$ and the traction-free inner boundary $\partial B_{\varepsilon}$, parameterized by the (small) inner radius $\varepsilon$.

Let us assume for simplicity that the center of the ring is located at origin of the coordinate system, and take polar coordinates $(r, \theta)$ with $\mathbf{e}_{r}$ pointing outwards and $\mathbf{e}_{\theta}$ perpendicularly in the counterclockwise direction. Then the displacement $u=u_{r} \mathbf{e}_{r}+u_{\theta} \mathbf{e}_{\theta}$ on the outer boundary $r=R$ is given in the form of a Fourier series:

$$
2 \mu\left(u_{r}+i u_{\theta}\right)=\sum_{k=-\infty}^{k=+\infty} U_{k} e^{i k \theta} .
$$

The boundedness of the boundary data in $H^{1 / 2}\left(\Gamma_{R}\right)$ translates (see Remark 2.4) into inequality (2.17) for the Fourier coefficients $U_{k}$.

The solution in $C(R, \varepsilon)$ must be compared with the solution in $C(R)$, so we use the complex variable method, described in [23,33], to construct the solutions.

Proposition A.1. For plane domains with a hole, the solutions of the elasticity 
boundary value problems take the form

$$
\begin{aligned}
\sigma_{r r}-i \sigma_{r \theta} & =2 \Re \phi^{\prime}-e^{2 i \theta}\left(\bar{z} \phi^{\prime \prime}+\psi^{\prime}\right), \\
\sigma_{r r}+i \sigma_{\theta \theta} & =4 \Re \phi^{\prime}, \\
2 \mu\left(u_{r}+i u_{\theta}\right) & =e^{-i \theta}\left(\kappa \phi-z \bar{\phi}^{\prime}-\bar{\psi}\right),
\end{aligned}
$$

where $\phi, \psi$ are given by complex series

$$
\begin{aligned}
& \phi=A \log (z)+\sum_{k=-\infty}^{k=+\infty} a_{k} z^{k}, \\
& \psi=-\kappa \bar{A} \log (z)+\sum_{k=-\infty}^{k=+\infty} b_{k} z^{k} .
\end{aligned}
$$

Here $\mu$ is the Lamé constant, $\nu$ the Poisson ratio, $\kappa=3-4 \nu$ in the plane strain case, and $\kappa=(3-\nu) /(1+\nu)$ for plane stress. In addition, $\Re \varphi$ is used to denote the real part of a complex function $\varphi$, while $\Im \varphi$ is going to be used to denote its imaginary counterpart.

Now, the displacement boundary condition for $r=R$ is substituted into the last line of (A.2) and, taking into account (A.3),

$$
\begin{aligned}
2 \mu\left(u_{r}+i u_{\theta}\right)= & 2 \kappa A r \log (r) \frac{1}{z}-\bar{A} \frac{1}{r} z \\
& +\sum_{p=-\infty}^{p=+\infty}\left[\kappa r a_{p+1}-(1-p) \bar{a}_{1-p} r^{-2 p+1}-\bar{b}_{-(p+1)} r^{-2 p-1}\right] z^{p},
\end{aligned}
$$

we obtain the infinite system of linear equations

$$
\begin{aligned}
& p=-1: \quad 2 \kappa A r \log (r)+\left(\kappa a_{0}-\bar{b}_{0}\right)-2 \bar{a}_{2} r^{2}=U_{-1}, \\
& p=1: \quad-\bar{A}+\kappa r^{2} a_{2}-\bar{b}_{-2} \frac{1}{r^{2}}=U_{1}, \\
& p \notin\{-1,1\}: \quad \kappa r^{p+1} a_{p+1}-(1-p) \bar{a}_{1-p} r^{-p+1}-\bar{b}_{-(p+1)} r^{-(p+1)}=U_{p} .
\end{aligned}
$$

The traction-free condition

$$
\sigma \mathbf{e}_{r}=\left[\sigma_{r r}, \sigma_{r \theta}\right]^{\top}
$$

on a circle means $\sigma_{r r}=\sigma_{r \theta}=0$. Hence, for $r:=\varepsilon$, we have another infinite system of linear equations,

$$
\begin{aligned}
& p=-1: \quad 2 A+2 \bar{a}_{2} r^{2}+2 \frac{1}{r^{2}} b_{-2}=0, \\
& p=1: \quad(\kappa+1) \frac{1}{r^{2}} \bar{A}=0, \\
& p \notin\{-1,1\}: \quad(1+p) a_{p+1}+\bar{a}_{1-p} r^{-2 p}+\frac{1}{r^{2}} b_{p-1}=0 .
\end{aligned}
$$

Define $d_{0}=\kappa a_{0}-\bar{b}_{0}$ since $a_{0}, b_{0}$ appear only in this combination. Using (A.4), we may recover the solution for the full circle. Because in this case the singularities must vanish, we have $b_{-k}=a_{-k}=A=0$ for $k=1,2, \ldots$, and after comparing the same 
powers of $r$, we find

$$
\begin{aligned}
& d_{0}^{0}=U_{-1}+\frac{2}{\kappa} \bar{U}_{1}, \quad \Re a_{1}^{0}=\frac{1}{(\kappa-1) R} \Re U_{0}, \quad \Im a_{1}^{0}=\frac{1}{(\kappa+1) R} \Im U_{0}, \\
& a_{k}^{0}=\frac{1}{\kappa R^{k}} U_{k-1}, \quad b_{k}^{0}=-\frac{1}{R^{k}}\left[(k+2) \frac{1}{\kappa} U_{k+1}+\bar{U}_{-(k+1)}\right], \quad k>1 .
\end{aligned}
$$

We use the same argument for the ring. Here singularities may be present, because the origin is not in the ring. Hence, from (A.4) with $r=R$ and (A.5) with $r=\varepsilon$, we obtain $A=0$ and the formulae

$$
\begin{aligned}
d_{0} & =A_{-1}+\frac{2 R^{4}}{\kappa R^{4}+\varepsilon^{4}} \bar{U}_{1}, & a_{2} & =\frac{R^{2}}{\kappa R^{4}+\varepsilon^{4}} U_{1}, \\
\Re a_{1} & =\frac{R}{(\kappa-1) R^{2}+2 \varepsilon^{2}} \Re U_{0}, & \Im a_{1} & =\frac{1}{\kappa+1} \Im A_{0}, \\
b_{-1} & =-\frac{2 \varepsilon^{2} R}{(\kappa-1) R^{2}+2 \varepsilon^{2}} \Re U_{0}, & b_{-2} & =-\frac{\varepsilon^{4} R^{2}}{\kappa R^{4}+\varepsilon^{4}} \bar{U}_{1} .
\end{aligned}
$$

The remaining part of the coefficients is computed later. However, we may at this stage compare the results with known solutions for the uniformly stretched circle or ring obtained in another way. In such a case $U_{0}=2 \mu u_{r}(R)$ does not vanish and, for the full circle, $\psi=0, \phi=a_{1}^{0} z$ with

$$
a_{1}^{0}=\frac{2 \mu}{(\kappa-1) R} u_{r}(R)
$$

For the ring we have $\phi=a_{1} z, \psi=b_{-1} \frac{1}{z}$, where

$$
a_{1}=\frac{1}{(\kappa-1)+2 \varepsilon^{2}} 2 \mu u_{R}(1), \quad b_{-1}=-\frac{2 \varepsilon^{2}}{(\kappa-1)+2 \varepsilon^{2}} 2 \mu u_{R}(1) .
$$

After substitutions we obtain, in both cases, the same results as given in [17]. Similarly the comparison with the solution for the ring with displacement conditions on both boundaries, obtained in [14] also using the complex method, confirms the correctness of our formulas.

There remains to compute the remaining part of coefficients $a_{k}, b_{k}$ for the ring. Taking $p=-k, k=2,3, \ldots$, the conditions on $\Gamma_{R}$ and $\partial B_{\varepsilon}$ lead to

$$
\begin{aligned}
\kappa a_{-(k-1)} R^{-(k-1)}-(k+1) \bar{a}_{k+1} R^{k+1}-\bar{b}_{k-1} R^{k-1} & =U_{-k}, \\
-(k-1) a_{-(k-1)} \varepsilon^{2}+\bar{a}_{k+1} \varepsilon^{2(k+1)}+b_{-(k+1)} & =0,
\end{aligned}
$$

while $p=+k, k=2,3, \ldots$, results in

$$
\begin{aligned}
\kappa a_{k+1} R^{k+1}+(k-1) \bar{a}_{-(k-1)} R^{-(k-1)}-\bar{b}_{-(k+1)} R^{-(k+1)} & =U_{k}, \\
(k+1) a_{k+1} \varepsilon^{2(k+1)}+\bar{a}_{-(k-1)} \varepsilon^{2}+b_{k-1} \varepsilon^{2 k} & =0 .
\end{aligned}
$$

These systems may be represented in a recursive form, convenient for numerical computations and further analysis. Namely,

$$
\left[\begin{array}{ll}
S_{k}(\varepsilon)_{11}, & S_{k}(\varepsilon)_{12} \\
S_{k}(\varepsilon)_{21}, & S_{k}(\varepsilon)_{22}
\end{array}\right]\left[\begin{array}{c}
a_{k+1} \\
b_{k-1}
\end{array}\right]=\left[\begin{array}{c}
U_{k} \\
\bar{U}_{-k}
\end{array}\right]
$$


where the entries $S_{k}(\varepsilon)_{i j}$ are given by

$$
\begin{aligned}
& S_{k}(\varepsilon)_{11}=\kappa R^{k+1}-\left(k^{2}-1\right) R^{1-k} \varepsilon^{2 k}+k^{2} R^{-(k+1)} \varepsilon^{2(k+1)}, \\
& S_{k}(\varepsilon)_{12}=-(k-1)\left(R^{1-k} \varepsilon^{2(k-1)}-R^{-(k+1)} \varepsilon^{2 k}\right), \\
& S_{k}(\varepsilon)_{21}=-(k+1)\left(R^{k+1}+\kappa R^{1-k} \varepsilon^{2 k}\right), \\
& S_{k}(\varepsilon)_{22}=-R^{k-1}-\kappa R^{1-k} \varepsilon^{2(k-1)},
\end{aligned}
$$

as well as by

$$
\left[\begin{array}{l}
a_{-(k-1)} \\
b_{-(k+1)}
\end{array}\right]=\left[\begin{array}{ll}
-(k+1) \varepsilon^{2 k}, & -\varepsilon^{2(k-1)} \\
-k^{2} \varepsilon^{2(k+1)}, & -(k-1) \varepsilon^{2 k}
\end{array}\right]\left[\begin{array}{l}
\bar{a}_{k+1} \\
\bar{b}_{k-1}
\end{array}\right] .
$$

In fact the formulae (A.10), (A.9) are correct also for $k=0,1$ and in the limit $\varepsilon \rightarrow 0^{+}$, but the derivation must separate these cases. Thus, for given $k>1$ and using some initial $a_{k}, b_{k}$ obtained earlier, we may first compute $a_{k+1}, b_{k-1}$ using (A.9) and then $a_{-(k-1)}, b_{-(k+1)}$ from (A.10).

We may now use the above results for the asymptotic analysis of the solution. To simplify the formulae, we assume $R=1$, which means only rescaling and does not diminish generality (in the general case, $\varepsilon$ would be replaced by $\varepsilon / R$ ). Then by direct computation we get the following bounds for the differences between the coefficients on the full circle and the ring: For the initial values of $k$, they read

$$
\begin{aligned}
& d_{0}-d_{0}^{0}=-\varepsilon^{4} \frac{2}{\kappa\left(\kappa R^{4}+\varepsilon^{4}\right)} \bar{U}_{1}, \\
& a_{1}-a_{1}^{0}=-\varepsilon^{2} \frac{2}{(\kappa-1) R\left((\kappa-1) R^{2}+2 \varepsilon^{2}\right)} \Re U_{0}, \\
& a_{2}-a_{2}^{0}=-\varepsilon^{4} \frac{1}{\kappa R^{2}\left(\kappa R^{4}+\varepsilon^{4}\right)} U_{1},
\end{aligned}
$$

and for higher values,

$$
\left|a_{3}-a_{3}^{0}\right| \leq \Lambda\left(\left|U_{2}\right| \varepsilon^{4}+\left|U_{-2}\right| \varepsilon^{2}\right)
$$

and for $k=4,5, \ldots$,

$$
\left|a_{k}-a_{k}^{0}\right| \leq \Lambda\left(\left|U_{k-1}\right| \varepsilon^{3(k-1) / 2}+\left|U_{1-k}\right| \varepsilon^{3(k-2) / 2}\right),
$$

where the exponent $k / 2$ has been used to counteract the growth of $k^{2}$ in terms like $k^{2} \varepsilon^{k / 2}$. Similarly

$$
\left|b_{1}-b_{1}^{0}\right| \leq \Lambda\left(\left|U_{2}\right| \varepsilon^{4}+\left|U_{-2}\right| \varepsilon^{2}\right),
$$

and for $k=2,3, \ldots$,

$$
\left|b_{k}-b_{k}^{0}\right| \leq \Lambda\left(\left|U_{k+1}\right| \varepsilon^{3(k+1) / 2}+\left|U_{-(k+1)}\right| \varepsilon^{3 k / 2}\right) .
$$

From relation (A.10) we get other estimates:

$$
\begin{aligned}
& \left|a_{-k}\right| \leq \Lambda \varepsilon^{2 k}\left(\left|U_{k+1}\right|+\left|U_{-(k+1)}\right|\right), \quad k=1,2, \ldots, \\
& \left|b_{-k}\right| \leq \Lambda \varepsilon^{2(k-1)}\left(\left|U_{k-1}\right|+\left|U_{1-k}\right|\right), \quad k=3,4, \ldots
\end{aligned}
$$

Here $\Lambda$ is a constant independent of $\varepsilon$ and $U_{i}$. Observe that the corrections proportional to $\varepsilon^{2}$ are present only in $a_{1}, b_{1}, a_{3}, b_{-1}, a_{-1}$. The rest is of the order at least $O\left(\varepsilon^{3}\right)$ (in fact, $O\left(\varepsilon^{4}\right)$ ). 


\section{REFERENCES}

[1] G. Allaire, F. Jouve, and N. Van Goethem, Damage and fracture evolution in brittle materials by shape optimization methods, J. Comput. Phys., 230 (2011), pp. 5010-5044.

[2] H. Ammari And H. Kang, Polarization and Moment Tensors with Applications to Inverse Problems and Effective Medium Theory, Appl. Math. Sci. 162, Springer-Verlag, New York, 2007.

[3] S. Amstutz And H. ANDRÄ, A new algorithm for topology optimization using a level-set method, J. Comput. Phys., 216 (2006), pp. 573-588.

[4] S. Amstutz, S. M. Giusti, A. A. Novotny, and E. A. De Souza Neto, Topological derivative for multi-scale linear elasticity models applied to the synthesis of microstructures, Internat. J. Numer. Methods Engrg., 8 (2010), pp. 733-756.

[5] S. Amstutz, A. A. Novotny, And E. A. De Souza Neto, Topological derivative-based topology optimization of structures subject to Drucker-Prager stress constraints, Comput. Methods Appl. Mech. Engrg., 233/236 (2012), pp. 123-136.

[6] E. Bänsch, M. Kaltenbacher, G. Leugering, F. Schury, and F. Wein, Optimization of electro-mechanical smart structures, in Constrained Optimization and Optimal Control for Partial Differential Equations, Internat. Ser. Numer. Math. 160, Birkhäuser/Springer Basel AG, Basel, 2012, pp. 501-519.

[7] R. C. Carbonari, E. C. N Silva, and S. Nishiwaki, Design of piezoelectric multiactuated microtools using topology, Smart Materials and Structures, 14 (2005), pp. 1431-1447.

[8] R. C. Carbonari, E. C. N. Silva, and S. Nishiwaki, Optimum placement of piezoelectric material in piezoactuator design, Smart Materials and Structures, 16 (2007), pp. 207-220.

[9] R. C. Carbonari, E. C. N. Silva, and G. H. Paulino, Multi-actuated functionally graded piezoelectric micro-tools design: A multiphysics topology optimization approach, Internat. J. Numer. Methods Engrg., 77 (2009), pp. 301-336.

[10] J. CÉa, S. Garreau, P. Gulllaume, and M. Masmoudi, The shape and topological optimizations connection, Comput. Methods Appl. Mech. Engrg., 188 (2000), pp. 713-726.

[11] P. G. Ciarlet, Mathematical Elasticity, Vol. I: Three-Dimensional Elasticity, North-Holland, Amsterdam, 1988.

[12] S. Garreau, P. Gulllaume, and M. Masmoudi, The topological asymptotic for PDE systems: The elasticity case, SIAM J. Control Optim., 39 (2001), pp. 1756-1778.

[13] N. Van Goethem And A. A. Novotny, Crack nucleation sensitivity analysis, Math. Methods Appl. Sci., 33 (2010), pp. 1978-1994.

[14] W. A. Gross, The second fundamental problem of elasticity applied to a plane circular ring, Z. Angew. Math. Phys., 8 (1957), pp. 71-73.

[15] M. Hintermüller AND A. LAURAin, Multiphase image segmentation and modulation recovery based on shape and topological sensitivity, J. Math. Imaging Vision, 35 (2009), pp. 1-22.

[16] M. Hintermüller, A. Laurain, ANd A. A. Novotny, Second-order topological expansion for electrical impedance tomography, Adv. Comput. Math., 36 (2012), pp. 235-265.

[17] M. Kachanov, B. Shafiro, and I. Tsukrov, Handbook of Elasticity Solutions, Kluwer Academic Publishers, Dordrecht, The Netherlands, 2003.

[18] I. LASIECKA, Mathematical Control Theory of Coupled PDEs, CBMS-NSF Regional Conf. Ser. in Appl. Math. 75, SIAM, Philadelphia, 2002.

[19] G. Leugering, A. A. Novotny, G. Perla-Menzala, and J. SokoŁowski, On shape optimization for an evolution coupled system, Appl. Math. Optim., 64 (2011), pp. 441-466.

[20] G. Leugering, A. A. Novotny, G. Perla-Menzala, and J. SokoŁowski, Shape sensitivity analysis of a quasi-eletrostatic piezoelectric system in multilayered media, Math. Methods Appl. Sci., 33 (2010), pp. 2118-2131.

[21] V. N. Melnik, Existence and uniqueness theorems for a generalized solution of a class of nonstationary problems of coupled electroelasticity, Soviet Math. (Iz. VUZ), 35 (1991), pp. $23-30$

[22] D. Mercier and S. Nicaise, Existence, uniqueness, and regularity results for piezoelectric systems, SIAM J. Math. Anal., 37 (2005), pp. 651-672.

[23] N. I. Muskhelishvili, Some Basic Problems on the Mathematical Theory of Elasticity, Noordhoff, Groningen, The Netherlands, 1952

[24] S. A. Nazarov and J. Sokotowski, The topological derivative of the Dirichlet integral under the formation of a thin bridge, Sibirsk. Mat. Zh., 45 (2004), pp. 410-426 (Russian); translation in Siberian Math. J., 45 (2004), pp. 341-355.

[25] S. A. Nazarov, A. S. SlutskiJ, And J. SokoŁOwski, Topological derivative of the energy functional due to formation of a thin ligament on a spatial body, Folia Math., 12 (2005), pp. 39-72. 
[26] S. A. Nazarov and J. SokoŁowski, Asymptotic analysis of shape functionals, J. Math. Pures Appl. (9), 82 (2003), pp. 125-196.

[27] A. A. Novotny and J. SokoŁowski, Topological Derivatives in Shape Optimization, Interact. Mech. Math., Springer, Berlin, 2013.

[28] S. Osher and J. A. Sethian, Front propagating with curvature dependent speed: Algorithms based on Hamilton-Jacobi formulations, J. Comput. Phys., 78 (1988), pp. 12-49.

[29] T. A. Poulsen, A simple scheme to prevent checkerboard patterns and one-node connected hinges in topology optimization, Struct. Multidisc. Optim., 24 (2002), pp. 396-399.

[30] E. C. N. Silva AND N. KiKUChi, Design of piezoelectric transducers using topology optimization, Smart Materials and Structures, 8 (1999), pp. 350-364.

[31] J. SokoŁowski AND A. Żochowski, On the topological derivative in shape optimization, SIAM J. Control Optim., 37 (1999), pp. 1251-1272.

[32] J. SokoŁowski AND A. Żochowski, Modelling of topological derivatives for contact problems, Numer. Math., 102 (2005), pp. 145-179.

[33] J. SokoŁowski AND A. Żochowski, Topological derivatives in plane elasticity, in Proceedings of the 23rd IFIP TC 7 Conference on System Modeling and Optimization, IFIP Adv. Inform. Commun. Tech. 312, Springer, Berlin, 2009, pp. 459-475.

[34] J. SokoŁowski And J.-P. Zolesio, Introduction to Shape Optimization-Shape Sensitivity Analysis, Springer, Berlin, 1992.

[35] T. Valent, Boundary Value Problems of Finite Elasticity, Tracts in Natural Philos. 31, Springer, Berlin, 1988. 